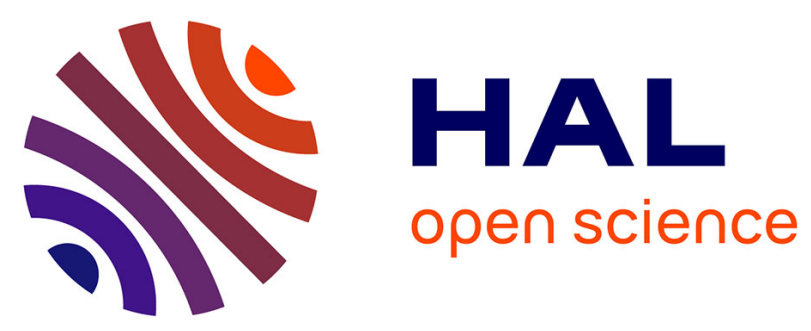

\title{
Uncoupling of IL-6 signaling and LC3-associated phagocytosis drives immunoparalysis during sepsis
}

Tonia Akoumianaki, Katerina Vaporidi, Eleni Diamantaki, Frédéric Pène,

Remi Beau, Mark S Gresnigt, Marina Gkountzinopulou, Maria Venichaki, Elias Drakos, Jamel El-Benna, et al.

\section{To cite this version:}

Tonia Akoumianaki, Katerina Vaporidi, Eleni Diamantaki, Frédéric Pène, Remi Beau, et al.. Uncoupling of IL-6 signaling and LC3-associated phagocytosis drives immunoparalysis during sepsis. Cell Host \& Microbe, 2021, 29 (8), pp.1277-1293.e6. 10.1016/j.chom.2021.06.002 . hal-03447781

\author{
HAL Id: hal-03447781 \\ https://hal.science/hal-03447781
}

Submitted on 24 Nov 2021

HAL is a multi-disciplinary open access archive for the deposit and dissemination of scientific research documents, whether they are published or not. The documents may come from teaching and research institutions in France or abroad, or from public or private research centers.
L'archive ouverte pluridisciplinaire HAL, est destinée au dépôt et à la diffusion de documents scientifiques de niveau recherche, publiés ou non, émanant des établissements d'enseignement et de recherche français ou étrangers, des laboratoires publics ou privés. 


\section{Uncoupling of IL-6 signaling and LC3-associated phagocytosis drives immunoparalysis during sepsis}

Tonia Akoumianaki, ${ }^{1}$ Katerina Vaporidi, ${ }^{2}$ Eleni Diamantaki, ${ }^{2}$ Frederic Pene, ${ }^{3}$ Remi Beau, ${ }^{4}$ Mark S. Gresnigt, ${ }^{5,6}$ Marina Gkountzinopulou, ${ }^{1}$ Maria Venichaki, ${ }^{7}$ Elias Drakos, ${ }^{8}$ Jamel ElBenna, 9 George Samonis, ${ }^{1}$ Kieu T.T. Le, ${ }^{5,10}$ Vinod Kumar, ${ }^{5,10}$ Dimitrios Georgopoulos, ${ }^{1}$ Frank L. van de Veerdonk, ${ }^{5}$ Mihai G. Netea, 5,11 Jean-Paul Latge, ${ }^{1,4}$ and Georgios Chamilos $1,12,13, *$

${ }^{1}$ Laboratory of Clinical Microbiology and Microbial Pathogenesis, School of Medicine, University of Crete, Voutes, 71110 Heraklion, Crete, Greece

${ }^{2}$ Department of Intensive Care Medicine, University Hospital of Heraklion, School of Medicine, University of Crete, Voutes, 71110 Heraklion, Crete, Greece

${ }^{3}$ Medical ICU, Hôpital Cochin, Hôpitaux Universitaires Paris Centre, Assistance Publique Hôpitaux de Paris, Institut Cochin INSERM U1016, CNRS UMR 8104, Université Paris Descartes, Paris, France

${ }^{4}$ Unité des Aspergillus, Institut Pasteur, Paris 75015, France

${ }^{5}$ Department of Internal Medicine (463) and Radboud Center for Infectious Diseases (RCI), Radboudumc, Geert Grooteplein 8, 6500 HB Nijmegen, the Netherlands

${ }^{6}$ Department of Microbial Pathogenicity Mechanisms, Leibniz Institute for Natural Product Research and Infection Biology - Hans-Knoell-Institute, Beutenbergstrasse 11a, 07745 Jena, Germany

${ }^{7}$ Laboratory of Clinical Chemistry, School of Medicine, University of Crete, Voutes, 71110 Heraklion, Crete, Greece

${ }^{8}$ Department of Pathology, School of Medicine, University of Crete, Voutes, 71110 Heraklion, Crete, Greece

${ }^{9}$ Université de Paris, Centre de Recherche sur l'Inflammation (CRI), INSERM U1149, CNRSERL 8252, Laboratoire d'Excellence Inflamex, Faculté de Médecine Xavier Bichat, Paris, France

${ }^{10}$ University of Groningen, University Medical Center Groningen, Department of Genetics, Groningen, the Netherlands

${ }^{11}$ Department for Genomics \& Immunoregulation, Life and Medical Sciences Institute (LIMES), University of Bonn, 53115 Bonn, Germany

${ }^{12}$ Institute of Molecular Biology and Biotechnology, Foundation for Research and Technology, 71300 Heraklion, Crete, Greece

*Correspondence: hamilos@imbb.forth.gr https://doi.org/10.1016/j.chom.2021.06.002 


\section{Highlights}

•ERK signaling regulates NADPH-oxidase mediated activation of LAP

-IL-6/JAK-2/Ninein axis controls microtubule-mediated ERK trafficking to the LAPosome

-Loss of IL-6 signaling inhibits LAP, leading to impaired pathogen killing

-IL-6 supplementation restores LAP defects and sepsis-induced immunoparalysis 


\section{Summary}

Immune deactivation of phagocytes is a central event in the pathogenesis of sepsis. Herein, we identify a master regulatory role of IL-6 signaling on LC3-associated phagocytosis (LAP) and reveal that uncoupling of these two processes during sepsis induces immunoparalysis in monocytes/macrophages. In particular, we demonstrate that activation of LAP by the human fungal pathogen Aspergillus fumigatus depends on ERK1/2-mediated phosphorylation of p47phox subunit of NADPH oxidase. Physiologically, autocrine IL-6/JAK2/Ninein axis orchestrates microtubule organization and dynamics regulating ERK recruitment to the phagosome and $\mathrm{LC}^{+}$phagosome (LAPosome) formation. In sepsis, loss of IL-6 signaling specifically abrogates microtubule-mediated trafficking of ERK, leading to defective activation of LAP and impaired killing of bacterial and fungal pathogens by monocytes/macrophages, which can be selectively restored by IL-6 supplementation. Our work uncovers a molecular pathway linking IL-6 signaling with LAP and provides insight into the mechanisms underlying immunoparalysis in sepsis.

\section{Keywords}

Aspergillus fumigatus, LAP, LC3-associated phagocytosis, phagosome, IL-6, JAK, NADPH oxidase, extracellular-signal-kinase regulated (ERK), microtubules, sepsis, sepsis, immunoparalysis 


\section{Introduction}

Sepsis, a complex and heterogeneous disease caused by a deregulated inflammatory response to an infectious insult, remains a leading cause of death worldwide (Reinhart et al., 2017; Singer et al., 2016; van der Poll et al., 2017). Numerous clinical trials on immunomodulation in sepsis have failed to improve patient outcome, emphasizing the need for deeper mechanistic insight on pathogenesis (Arnold, 2018; Peters van Ton et al., 2018; Rello et al., 2018). In recent years, it has been realized that many patients who survive the initial sepsis episode enter a prolonged state of immune deactivation, termed sepsis-induced immunoparalysis, accounting for treatment failures and death due to secondary infections by opportunistic pathogens (Boomer et al., 2011; Hotchkiss et al., 2013).

As opposed to "immunological tolerance," a physiological compensatory mechanism that confers a balanced inflammatory response to an infectious agent, sepsis-induced immunoparalysis is characterized by impaired activation of essential immune effector pathways in myeloid phagocytes (Cheng et al., 2016; Domínguez-Andrés et al., 2019; van der Poll et al., 2017) and loss of their microbicidal activity (Boomer et al., 2011; Chiswick et al., 2015; Döcke et al., 1997; Hotchkiss et al., 2013; van der Poll et al., 2017). At the molecular level, supra-physiological induction of pathways regulating "immunological tolerance," results in cytokine hyporesponsiveness and is a central molecular event of immune deactivation in sepsis (Hotchkiss et al., 2013; van der Poll et al., 2017). In particular, ROS-mediated super-induction of activating transcription factor 3 (ATF3) suppress innate cytokines, predominantly IL-6, and accounts for heightened susceptibility to secondary infection by opportunistic bacterial and fungal pathogens (Hoetzenecker et al., 2011). Aberrant ATF3 expression in sepsis is also induced via metabolic reprogramming of myeloid phagocytes (Bambouskova et al., 2018; Domínguez-Andrés et al., 2019). Furthermore, epigenetic modulation of myeloid progenitors induced by sepsis leads to persistent immune deactivation of professional phagocytic cells (Wen et al., 2008; Zhang et al., 2016). Immunotherapy with cytokines, such as IFN- $\gamma$ and GM-CSF, partially reverses cytokine hypo-responsiveness and restores the microbicidal activity of sepsis monocytes (Arnold, 2018; Döcke et al., 1997; Leentjens et al., 2012; Peters van Ton et al., 2018; van der Poll et al., 2017). However, successful implementation of cytokine therapies in human patients with sepsis requires understanding the underlying signaling defects that account for immunoparalysis in the individual patient.

LC3 associated phagocytosis (LAP), a phagosome biogenesis pathway utilizing part of the autophagy machinery in response to certain pattern-recognition receptors (PRRs), promotes phagolysosomal fusion and killing of an expanding list of pathogens by macrophages (Heckmann and Green, 2019; Martinez et al., 2015; Sanjuan et al., 2007). Besides, LAP regulates the antiinflammatory activity of macrophages (Chu et al., 2016; Heckmann and Green, 2019; Heckmann et al., 2019; Martinez et al., 2011, 2016). LAP utilizes a distinct signaling pathway from canonical autophagy and is regulated by Rubicon (Martinez et al., 2015). In particular, NADPHoxidase-complex-mediated ROS production is a fundamental requirement for $\mathrm{LC}^{+}$phagosome (LAPosome) formation (Martinez et al., 2015).

Currently, there is no single biological marker to define sepsis immunoparalysis, and the underlying molecular mechanisms remain at least in part obscure. Aspergillus fumigatus, an airborne saprophytic mold shaping immunological tolerance in human respiratory epithelia 
(Bacher et al., 2016), has become a model pathogen to study immune deactivation in sepsis (Benjamim et al., 2003, 2005; Hoetzenecker et al., 2011). Interestingly, life-threatening respiratory infections caused by this opportunistic fungus are increasingly encountered in patients recovering from bacterial and viral sepsis (Bae et al., 2020; Bartoletti et al., 2020; Bassetti et al., 2017; Colombo et al., 2017; Dimopoulos et al., 2003; Meersseman et al., 2004). We and others have shown the crucial role of LAP in protective immunity against Aspergillus and other human pathogens (Akoumianaki et al., 2016; Kyrmizi et al., 2018, 2013; Martinez et al., 2015; Oikonomou et al., 2016), (de Luca et al., 2014). However, the molecular mechanisms of crosstalk of cytokine signaling and LAP are poorly understood. In addition, the role of LAP in the pathogenesis of sepsis is currently unknown.

Using Aspergillus as a model pathogen, we identify defective activation of LAP as a hallmark feature of immune deactivation of phagocytes in septic patients and mice. In particular, we discover a unique role of autocrine IL-6/JAK2/Ninein axis on the regulation of microtubuledependent ERK recruitment to the phagosome for LAPosome formation. Next, we demonstrate that loss of IL-6 signaling during sepsis selectively disrupts microtubule dynamics regulating ERK-dependent activation of LAP, leading to impaired control of bacterial and fungal pathogens by monocytes/macrophages. IL-6 specifically restores microbicidal activity of myeloid phagocytes in sepsis patients and mice, in a LAP-dependent way. Collectively, our findings link IL-6 signaling with LAP, with an important role in the development of immunoparalysis of sepsis, which can be explored as a therapeutic target.

\section{Results}

\section{Defective activation of LAP in monocytes is a dominant feature of immunoparalysis in human patients with sepsis}

Given the essential role of LAP in killing of Aspergillus by phagocytes (Akoumianaki et al., 2016; Kyrmizi et al., 2013, 2018; Martinez et al., 2015), we explored whether this pathway is modulated by sepsis. We obtained monocytes from 38 consecutive patients admitted to intensive care unit (ICU) with community-acquired septic shock. Functional studies were performed on the day of patient admission (day 1) and upon recovery from the infectious episode (day 7) (Figure 1A). We used melanin-deficient (albino) conidia of Aspergillus fumigatus $\Delta p k s P$ mutant as model bioparticles that induce a robust activation of LAP (Akoumianaki et al., 2016; Kyrmizi et al., 2013, 2018; Martinez et al., 2015) and compared immune responses of monocytes from sepsis patients with those induced in monocytes of healthy individuals (controls). Importantly, killing of wild-type Aspergillus conidia is also dependent on activation of LAP upon cell wall melanin removal inside monocytes/macrophages (Akoumianaki et al., 2016; Kyrmizi et al., 2013, 2018; Martinez et al., 2015). Therefore, we additionally assessed killing of the isogenic wild-type A. fumigatus clinical isolate (ATCC46645) by monocytes of sepsis patients versus controls. In parallel, we analyzed clinical and microbiological characteristics and the outcome of patients to explore whether classical features of sepsis immunoparalysis (e.g., secondary infections) are associated with impaired LAP responses (Tables S1-S3). LAP was assessed by counting the percentage of $\mathrm{LC}^{+}$Aspergillus-containing phagosomes in monocytes by confocal microscopy (Figure S1) (Akoumianaki et al., 2016; Kyrmizi et al., 2013, 2018; Martinez et al., 2015). LAP responses following sepsis recovery (day 7; when patients are at increased risk for development of secondary infections) segregated patients in two distinct groups (Figure S1). When compared 
with healthy individuals $(n=19)$, group A included patients with intact LAP responses $(n=15$, $\mathrm{P}=\mathrm{NS}$ versus healthy control group), and group B included patients with defective activation of LAP $(n=23, p<0.0001$ versus healthy control group and versus patients with intact LAP) (Figure 1B). These differences in LAP responses were less apparent on day 1 of sepsis (Figure S1). Because LAP is dependent on NADPH oxidase (Martinez et al., 2015), we assessed NADPH oxidase activation in monocytes of sepsis patients by measuring p47phox translocation to the phagosome membrane. Notably, monocytes from patients with LAP defects displayed impaired recruitment of p47phox to the phagosome (Figures 1C and S1). Accordingly, we found evidence of phagosome maturation arrest in monocytes of patients with LAP defects following Aspergillus infection, suggested by the lack of acquisition of CD63 (a marker of phagolysosomal fusion and acidification) (Meersseman et al., 2004), (Figures 1D and S1).

Defective LAP responses in monocytes of patients recovering from sepsis (day 7) were significantly associated with impaired killing of conidia of a clinical isolate of Aspergillus (ATCC 46645) (Figures 1E and S1), and our melanin-deficient Aspergillus mutant used as a model for phagocytosis (Figure S1). In contrast, monocytes from patients with compromised LAP responses had no apparent defects on the level of phagocytosis of conidia of melanindeficient $\Delta p k s P$ Aspergillus strain (Figure S1). In addition, there was no difference in the percentage of $\mathrm{CD}_{16} 6^{+}$monocytes and the level of CD163 expression between patients with intact versus defective LAP responses (Figure S2). Of interest, HLA-DR expression were decreased in LAP-defective monocytes (Figure S2).

An impaired cytokine response of monocytes following ex vivo re-stimulation with TLR agonists is a prominent feature of immune deactivation in sepsis (Hotchkiss et al., 2013; van der Poll et al., 2017). Specifically, suppressed IL-6 production has a major causative role in the development of sepsis-induced immunoparalysis (Cenci et al., 2001; Hoetzenecker et al., 2011). Monocytes of patients with LAP defect on day 7 had a significant decrease of IL-6 production following overnight stimulation with A. fumigatus when compared with control healthy monocytes and monocytes of patients with intact LAP responses, which was proportional to the severity of the underlying LAP defect (Figure 1F; Table S2); production of other proinflammatory cytokines was decreased in monocytes of patients with LAP defects versus those with intact LAP but was not statistically significant (Figure S2).

Development of secondary infections is a hallmark clinical feature of sepsis-induced immunoparalysis (Boomer et al., 2011; Hotchkiss et al., 2013; van der Poll et al., 2017). Notably, patients with LAP defect had a higher clinical severity of sepsis on the day of admission in the ICU (APACHE II score) (Figure 1G) and a significant 3-fold increase in cumulative risk for secondary infections as compared with patients with intact LAP (Figure 1H; Tables S1-S3). Collectively, defective activation of LAP in monocytes of patients with sepsis is associated with classical immunological and clinical features of immunoparalysis.

\section{Localized ERK1/2 signaling on the phagosome regulates NADPH oxidase-dependent activation of LAP and is inhibited in sepsis}


Impaired phagosomal localization of p47phox was a striking upstream signaling defect in monocytes/macrophages of sepsis patients with a LAP defect (Figures 1B and 1C). To gain further insights into defective LAP activation in sepsis, we explored the signaling pathway regulating p47phox phosphorylation. We observed that infection of monocytes with Aspergillus conidia induced early and selective phosphorylation of p47phox on Ser345 (Figures 2A and S3); in contrast, PMA, a classical PKC activator, induced the phosphorylation of p47phox on distinct serine sites (Ser315, Ser328) (Figures 2A and S3). Since phosphorylation of p47phox on Ser345 is regulated by ERK1/2 signaling in human neutrophils (Boussetta et al., 2010; Dang et al., 2006; El-Benna et al., 2009; Makni-Maalej et al., 2013), we evaluated the role of ERK1/2 signaling on NADPH oxidase-mediated activation of LAP by Aspergillus in monocytes. Importantly, ERK1/2 signaling in monocytes was rapidly activated within minutes of infection by Aspergillus (Figures 2B and S3). In addition, immunostaining revealed an endosomal pattern of distribution of ERK in monocytes, with early and sustained ERK recruitment and phosphorylation on the phagosome following Aspergillus infection (Figures 2C and S3). Importantly, inhibition of ERK1/2 phosphorylation upon monocyte treatment with UO126 (Figures 2AB and S3), a specific MEK1/2 inhibitor (Favata et al., 1998), significantly reduced p47phox phosphorylation at Ser345 (Figure 2B), p47phox recruitment to the phagosome (Figure 2D), NADPH oxidase complex assembly - as demonstrated by inhibition of phagosomal localization of p22phox membrane subunit (Figure 2E) - intracellular ROS production (Figure 2F), and LAPosome formation (Figure 2G). Accordingly, inhibition of ERK1/2 signaling significantly decreased the killing of Aspergillus conidia by monocytes (Figure $2 \mathrm{H}$ ). Importantly, pharmacological inhibition of Class III PI3K (VPS34) complex by wortmannin did not impair ERK recruitment to the phagosome (Figure S3). Collectively, these studies reveal an essential role of ERK signaling in NADPHoxidase-dependent activation of LAP by Aspergillus. Monocytes of two representative patients recovering from sepsis with LAP defect (Figure 2I) had impaired trafficking of p47phox to the phagosome (Figure 2J) and blockade on ERK-dependent phosphorylation of p47phox at Ser345 (Figures $2 \mathrm{~K}$ and S3). Accordingly, ERK trafficking to the phagosome was impaired in patients with LAP defect compared with a healthy control (Figure 2K); while ERK1/2 phosphorylation was not always impaired (Pt-2; Figure S3). Collectively, these findings reveal a pronounced defect in ERK recruitment to the phagosome of sepsis patients with impaired activation of LAP.

\section{Autocrine IL-6 signaling regulates ERK trafficking and LAPosome formation independent of ERK1/2 phosphorylation}

IL-6 production is defective in phagocytes during immunoparalysis in sepsis (Hoetzenecker et al., 2011). We observed that defective IL-6 production in sepsis monocytes was significantly associated with LAP blockade (Figure 1F; Table S2). In contrast, inhibition of LAP was not associated with reduction in the expression of genes regulating ERK-dependent activation of LAP (Data S1). To explore the role of IL-6 on regulation of LAP, we evaluated phagosome responses in bone-marrow-derived macrophages (BMDMs), of IL-6 knockout (IL- $6^{-/}$) and control $\left(\right.$ IL- $6^{+/+}$) mice infected with Aspergillus; BMDMs are typically used for studies of LAP in myeloid phagocytes of mice (Akoumianaki et al., 2016; Kyrmizi et al., 2013, 2018; Martinez et al., 2015; Oikonomou et al., 2016), (de Luca et al., 2014). We observed that NADPH-oxidasemediated activation of LAP was significantly impaired in IL- $6^{-/-}$BMDMs as compared with control IL- $6^{+/+}$BMDMs. In particular, there was a significant decrease on localization of the 
membrane-associated (p22phox) (Figure 3A) and cytosolic (p47phox) (Figure 3B) subunits of NADPH oxidase complex to the phagosome, and defective LAPosome formation in the IL- $6^{-/}$ versus control (IL- $6^{+/+}$) BMDMs (Figure 3C). In addition, IL- $6^{-/-}$BMDMs had significant defects in acquisition of markers of phagosome acidification (Figure 3D), phagolysosomal fusion (Figures $3 \mathrm{E}$ and $3 \mathrm{~F}$ ), and in the ability to kill conidia of a wild-type clinical isolate of Aspergillus fumigatus (Figure 3G). Accordingly, pulmonary infection of $\mathrm{IL6}^{-/-}$mice with wild-type Aspergillus conidia resulted in significant increase in fungal load in the lungs as compared with infection of control IL6 ${ }^{+/+}$mice (Figures $3 \mathrm{H}$ and S4), without evidence of invasive fungal disease in histopathology (Figure S4).

Next, we assessed activation of ERK1/2 signaling by Aspergillus in BMDMs of IL- $6^{-/-}$versus IL- $6^{+/+}$mice. Of interest, western blot analysis of protein lysates obtained from BMDMs of IL$6^{-/-}$versus IL- $6^{+/+}$revealed no appreciable differences in total ERK1/2 levels and the degree of ERK1/2 phosphorylation induced by Aspergillus (Figures 3I and 3J). In sharp contrast, we found a significant defect in ERK1/2 phosphorylation and recruitment on Aspergillus-containing phagosomes of IL- $6^{-/-}$versus IL- $6^{+/+}$BMDMs (Figure $3 \mathrm{~K}$ ), which was rapidly restored within 15 min of IL-6 supplementation in culture media (Figure 3L). Furthermore, in contrast to other cytokines, IL-6 supplementation selectively enhanced defective killing of Aspergillus conidia by IL-6 ${ }^{-/}$BMDMs (Figure 3M). Collectively, these studies reveal a previously uncharacterized mechanism of action of IL-6 signaling on ERK trafficking to the phagosome and LAPosome formation.

\section{Defects in autocrine IL-6/JAK2/Ninein axis disrupt microtubule dynamics regulating phagosomal trafficking of ERK and LAPosome formation}

Next, we evaluated the mechanism of regulation of phagosomal trafficking of ERK by IL-6 signaling. The phagosome maturation process is regulated by microtubule dynamics (Blocker et al., 1997; Harrison et al., 2003). In particular, physical clustering of dynein motors into lipid micro-domains on the phagosome membrane leads to rapid directed transport of the phagosome toward microtubule minus ends to promote fusion with lysosomes and pathogen degradation (Rai et al., 2016). Therefore, we assessed whether the process of ERK recruitment to the phagosome is also regulated by microtubules. We found that the disruption of microtubule assembly via depolymerization induced by nocodazole or inhibition of microtubule dynamics following treatment of BMDMs with the microtubule-stabilizing agent taxol resulted in abolished ERK localization to Aspergillus-containing phagosomes (Figure 4A). Therefore, we specifically assessed the effect of IL- 6 on organization of the microtubule network and phagosome transport. Notably, immunostaining of uninfected $\mathrm{IL}-6^{-/-}$BMDMs for $\beta$-tubulin revealed major defects in the size of the microtubule organizing center (centrosome) at the steady state, as compared with control IL- $6^{+/+}$BMDMs, a defect that was reversed within 15 min of IL-6 supplementation in IL$6^{-/-}$BMDMs (Figure 4B). We also found that centripetal transport of Aspergillus-containing phagosomes from the cell periphery to the microtubule minus end (microtubule organization center) was inhibited in IL- $6^{-/-}$BMDMs, as compared with control IL- $6^{+/+}$BMDMs, a defect that was restored within minutes of IL-6 supplementation (Figure 4C-4E; Videos S1 and S2). Importantly, IL-6 ${ }^{-/}$BMDMs had no apparent defect in phagocytosis of different pathogens, including Aspergillus and Pseudomonas, and biotin beads (Figure S5). 
To investigate the signaling pathway of IL-6-depended regulation of microtubule dynamics and phagosome trafficking we evaluated the effect of inhibition of JAK/STAT signaling pathway downstream of IL-6 receptor on phagosomal localization of ERK, phagosome movement and LAP. Of interest, inhibition of JAK2 with Ruxolitinib, a specific JAK1/2 inhibitor (QuintásCardama et al., 2010) resulted in a significant, dose-dependent inhibition of phagosome transport toward the microtubule minus end (Figure 4F), leading to blockade in phagosomal localization of ERK (Figure 4G), LAPosome formation (Figure 4H) and fungal killing (Figure 4I). In contrast, inhibition of the phosphorylation of STAT3, which is downstream of JAK2, with the use of a specific STAT3 inhibitor (S3I-201) (Siddiquee et al., 2007) had no apparent effect on ERK trafficking to the phagosome (Data S2).

To further gain mechanistic insight on regulation of microtubule organizing center by IL-6/JAK2 signaling, we explored $\gamma$-tubulin expression, a master regulator of the centrosome nucleation, in IL-6 ${ }^{-/}$BMDMs. We found no differences in $\gamma$-tubulin perinuclear localization in the area of centrosomes of IL- $6^{+/+}$versus IL- $6^{-/-}$BMDMs by immunostaining (Figures $4 \mathrm{~J}$ and $4 \mathrm{~K}$ ). Next, we focused on the pattern of perinuclear localization of Ninein, a master regulatory protein of microtubule anchoring to the centrosome that has been shown to be phosphorylated by JAK2 (Jay et al., 2015). Of interest, we found a significant decrease in perinuclear expression of Ninein in IL-6 KO BMDMs, accompanied by loss of co-localization of ninein with JAK2, which was rapidly restored within min of IL-6 supplementation (Figures $4 \mathrm{~L}$ and $4 \mathrm{M}$ ). Collectively, these findings demonstrate a previously uncharacterized role of the pathway IL-6/JAK2/Ninein signaling on microtubule cytoskeleton dynamics, which then regulates phagosome transport and ERK-mediated LAPosome formation.

\section{Sepsis-induced susceptibility to fungal infections is associated with LAP blockade in mouse monocytes/macrophages}

To further explore the underlying mechanisms of LAP defect in sepsis, we developed a physiologically relevant mouse model of polymicrobial sepsis (peritonitis) by inducing different degrees of disease severity (mild sepsis, <5\% mortality rates; severe sepsis, $>50 \%$ mortality rates) (Figures 5A and 5B). Severe sepsis selectively compromised the ability of mice to control superinfection with Aspergillus, as evidenced by a significantly higher fungal load in the lungs of mice recovering from severe sepsis (day 7) (Figure 5C), when compared with mice recovering from mild sepsis or SHAM-operated mice; Aspergillus infection in mice recovering from severe sepsis (hereafter sepsis) was specifically associated with extensive inflammatory immunopathology (Figure S6) and invasive fungal growth (Figures 5D and S6). In line with the in vivo studies, defective activation of LAP following Aspergillus infection was induced in splenic monocytes, BMDMs, and alveolar macrophages (AMs) of mice recovering from sepsis, in contrast to SHAM-operated mice (Figures 5E, 5F, and S7). Furthermore, sepsis-induced LAP defect in BMDMs was associated with diminished ROS production in response to Aspergillus (Figure 5G) and phagosome maturation arrest, as evidenced by (1) defective accumulation of fluorescein isothiocyanate-labeled dextran (FITC-Dextran) to the phagosome following labeling of lysosomes (Figures 5H and 5I), (2) impaired phagosome acidification assessed by VATPase staining (Figure 5J), and (3) defective phagosomal localization of the lysosomal markers cathepsin-D (Figure 5K) and LAMP-1 (Figures 5L and S7). Finally, sepsis BMDMs displayed significant loss of fungicidal activity against conidia of wild-type Aspergillus (Figure 5M) and diminished IL-6 production upon re-stimulation with fungal conidia (Figure S7). Overall, these 
findings reveal that sepsis-induced immune deactivation in murine monocytes/macrophages is associated with defective activation of LAP.

\section{Loss of IL-6 signaling in mice and human patients with sepsis selectively impairs ERK trafficking, LAPosome formation, and fungal killing}

Next, we assessed whether LAP blockade in sepsis macrophages is mechanistically linked with defects in IL-6-dependent trafficking of ERK to the phagosome. We found a profound defect in centrosome organization and microtubule assembly at the baseline in BMDMs obtained from mice with severe sepsis, a phenotype reminiscent of microtubule cytoskeleton abnormalities in $\mathrm{IL}^{-6}{ }^{-1-}$ BMDMs (Figure 6A). Indeed, sepsis-induced defects in the size of microtubule organizing center in uninfected BMDMs were rapidly restored following IL-6 supplementation (Figure 6A). Accordingly, BMDMs obtained from mice with severe sepsis displayed significant defects on phagosome transport following infection with Aspergillus, which were restored within minutes of IL-6 treatment (Figure 6B).

In line with these results, ERK trafficking to Aspergillus-containing phagosomes was abolished in BMDMs of mice recovering from severe sepsis in contrast to control SHAM treated mice (Figures 6C and 6D). Instead, impaired phagosomal ERK recruitment was not always accompanied by defective ERK phosphorylation by Aspergillus (Data S2). Of interest, IL-6 supplementation resulted in complete restoration of phagosomal localization of ERK (Figures 6C and 6D) and LAPosome formation (Figure 6E) in BMDMs obtained from mice with severe sepsis. Furthermore, as opposite to other cytokines (TNF, IFN- $\gamma$ ), IL-6 treatment selectively enhanced defective killing of Aspergillus by sepsis BMDMs (Figure 6F).

Similar to the findings in macrophages of mice following sepsis recovery, we found defects in microtubule anchoring and organization at the centrosome and microtubule-mediated phagosome transport in monocytes of patients with sepsis-induced inhibition of LAP (Figure 6G). Importantly, IL-6 supplementation in culture media of human monocytes with sepsis-induced inhibition of LAP fully restored defective killing activity against Aspergillus (Figure 6H); instead, IL-6 treatment had no effect in fungicidal activity of healthy control monocytes. These studies reveal a previously uncharacterized role of IL-6 signaling on ERK-mediated activation of LAP in monocyte/macrophage with direct pathophysiological and therapeutic implications for immunoparalysis in sepsis.

\section{IL-6-induced restoration of microbicidal activity of myeloid phagocytes in sepsis depends on LAP}

In order to establish a causative relationship between loss of IL-6 signaling, LAP blockade, and sepsis immunoparalysis, we explored the effect of IL-6 supplementation on microbicidal activity of sepsis macrophages in mice with genetic defect of Atg5. BMDMs from mice with genetic deletion of Atg5 (LysMCre ${ }^{+/+}$; Atg5flox/flox mice; hereafter Atg $5^{-/}$BMDMs), an autophagy protein crucial for LAPosome formation (Akoumianaki et al., 2016; Martinez et al., 2015), had no apparent defects on microtubule cytoskeleton organization (Figure 7A); however, Atg $5^{-/}$ BMDMs displayed broad defects on phagosome biogenesis, comparable with those induced by 
severe sepsis (Figures 7B-7E). Notably, IL-6 supplementation resulted in full reversal of sepsisinduced defects in killing of Aspergillus conidia by Atg $5^{+/+}$BMDMs but failed to restore killing defects in Atg5 $5^{-/-}$BMDMs following sepsis (Figure 7F).

Next, we evaluated the physiological importance of IL-6/LAPosome pathway in macrophage antimicrobial activity against Pseudomonas, a bacterial pathogen classically associated with secondary infections due to sepsis-induced immunoparalysis (Boomer et al., 2011; van der Poll et al., 2017) including our patient cohort (Table S1). We initially verified that killing of a reference clinical isolate of Pseudomonas aeruginosa by macrophages depends on IL-6 signaling (Figure 7G) and LAP (Figure 7H). Next, we tested the effect of IL-6 supplementation on bactericidal capacity of BMDMs differentiated from control versus LAP-defective mice recovering from severe sepsis. Importantly, IL-6 supplementation significantly improved killing of Pseudomonas by BMDMs obtained from Atg $5^{+/+}$(control) BMDMs following recovery from severe sepsis (Figure 7I). In contrast, IL-6 failed to restore bactericidal activity of Atg5 $5^{-/-}$ BMDMs following sepsis (Figure 7I). Collectively, these studies lead to a new mechanism of regulation of LAP by IL-6 signaling with physiological importance in pathogenesis of sepsis immunoparalysis.

\section{Discussion}

Integration of signals from PRRs and cytokines to phagocytosis has a crucial role in successful control of infection by macrophages (Pauwels et al., 2017). In particular, PRR-mediated activation of the LAP pathway regulates phagosome maturation and confers protective immunity against a range of pathogens (Akoumianaki et al., 2016; Heckmann and Green, 2019; Kyrmizi et al., 2013, 2018; Martinez et al., 2015). Likewise, specific cytokines transcriptionally induce the expression of antimicrobial effectors to enhance the microbicidal capacity of macrophages (Forbester et al., 2018; Jung and Robinson, 2014; Shen et al., 2018; Subramanian Vignesh et al., 2013; Via et al., 1998). Moreover, reciprocal interactions of cytokines and LAP are required for an optimal anti-inflammatory function of macrophages (de Luca et al., 2014; Oikonomou et al., 2016). However, a mechanistic link between cytokine signaling and LAP has not yet been identified.

Herein, we employ sepsis as a model human disease to explore physiologically important interactions of cytokine signaling and LAP. Our studies in humans and mice "identify LAP blockade in phagocytes as a core immunological feature of sepsis-induced immunoparalysis." Moreover, we discover "a role for the autocrine IL-6/JAK2 axis in microbicidal activity of phagocytes via regulation of LAP, with direct implications in immune deactivation of sepsis."

In our initial studies, we found that defective LAP is a prominent feature of phagocyte dysfunction in patients recovering from sepsis, who display all classical features of immunoparalysis (Hotchkiss et al., 2013; van der Poll et al., 2017). In view of the absence of biological measures of immunoparalysis induced by sepsis, inhibition of LAP should be regarded as an immunological feature of this pathological process. Apart from monocytes/macrophages, neutrophils are major effectors of innate immune response in sepsis (Hotchkiss et al., 2013; van 
der Poll et al., 2017). Therefore, the role of LAP defects in immune deactivation of neutrophils in sepsis should be investigated.

We also identified a selective mechanism of p47phox phosphorylation at Ser345 in monocytes during fungal infection, a phosphorylation site regulated by MAPK signaling (Dang et al., 2006; El-Benna et al., 2009; Makni-Maalej et al., 2013). This reveals a major role of ERK1/2 signaling on NADPH oxidase-mediated activation of LAP both physiologically and in sepsis, independently of VPS-34 complex formation on the phagosome. Because cytokine deregulation has a major role in the pathogenesis of sepsis (van der Poll et al., 2017), we evaluated the direct role of cytokine signaling on ERK-dependent activation of LAP. We focused on IL-6, a signature cytokine of sepsis-induced immunosuppression and related susceptibility to aspergillosis (Cenci et al., 2001; Hoetzenecker et al., 2011). Surprisingly, we observed that although p47phoxmediated activation of NADPH oxidase was abolished in IL-6 knockout (KO) BMDMs during fungal infection, upstream ERK1/2 phosphorylation remained intact. In contrast, ERK1/2 recruitment on the phagosome was abolished in IL-6 KO BMDMs, which explained the downstream blockade in NADPH oxidase-dependent activation of LAP. These findings confirmed our hypothesis on distinctive pathways regulating ERK phosphorylation and trafficking on the phagosome and revealed a central regulatory role for IL-6 signaling on endosomal trafficking of ERK and LAPosome formation.

Importantly, we discovered that IL-6 KO BMDMs display profound disorganization of microtubule organizing center at steady state, associated with impaired LAP-dependent killing of pathogens without an apparent defect in phagocytosis. Subsequently, we found that microtubuledependent phagosome movement and ERK trafficking were regulated by JAK2, independently of STAT3. Of interest, both STAT3 (Jay et al., 2015; Ma and Sayeski, 2007) and JAK2 (Morris et al., 2017; $\mathrm{Ng}$ et al., 2006) are involved in microtubule organization dynamics via interactions with distinct signaling pathways. Evermore, JAK2 phosphorylates and is negatively regulated by the centrosomal protein ninein (Jay et al., 2015). In particular, Ninein is a master regulator of microtubule anchoring to the centrosome. Accordingly, inhibition of JAK2 has been shown to selectively impair the size of microtubule organizing center without affecting the process of centrosome nucleation, a function regulated by $\gamma$-tubulin (Jay et al., 2015). Indeed, our findings on diminished Ninein expression and co-localization with JAK2 in perinuclear regions of IL-6 KO macrophages without an apparent defect in topology of $\gamma$-tubulin are in line with previous studies (Ma and Sayeski, 2007; Rai et al., 2016; Via et al., 1998).

In proof-of-concept studies, we demonstrated that monocytes/macrophages isolated from septic conditions display defects in microtubule cytoskeleton organization, ERK trafficking, LAPosome formation, and pathogen killing. These are specifically related to loss of IL- 6 signaling, as they were all restored within minutes of IL-6 supplementation. Furthermore, we revealed that IL-6 induced restoration of LAP defects in sepsis has broad implications in phagocyte immunity against major bacterial and fungal pathogens. Of interest, previous studies report on the restoration of sepsis-induced immune deactivation in monocytes following several days of immunotherapy with IFN- $\gamma$ (Döcke et al., 1997). It would be important to investigate the effects of prolonged immunotherapy with IFN- $\gamma$ on IL-6/LAP axis. Alternatively, other cytokines may regulate phagosome biogenesis or trigger canonical autophagy (e.g., xenophagy) responses against certain microbial pathogens (e.g., intracellular bacteria) independently of NADPH oxidase signaling and LAP (Sokolovska et al., 2013). In addition, whether unique intracellular 
trafficking of IL-6 as compared with other cytokines may have a role on the specialized role of this cytokine on regulation of ERK-dependent activation of LAP deserves exploitation (Murray et al., 2005). Collectively, our study identifies an unexplored physiological pathway linking autocrine IL-6/JAK2/Ninein axis, cytoskeleton organization, and LAP with the microbicidal activity of myeloid phagocytes and offers mechanistic insight in immunoparalysis of sepsis.

Our findings have also broad implications for understanding emerging mechanisms of iatrogenic immunosuppression predisposing to opportunistic infections. In particular, recent clinical studies report an alarming increase in cases of severe bacterial and fungal diseases in patients receiving small molecule kinase inhibitors (SMKIs), in the absence of additional immunosuppressive conditions. Notably, SMKIs targeting ERK1/2 and JAK/STAT signaling have been recently associated with clinical cases of invasive aspergillosis (Chamilos et al., 2018a, 2018b; Dioverti et al., 2018; Lussana et al., 2018). Preclinical studies also demonstrate compromised killing capacity of phagocytes against several pathogens, including Aspergillus, following pharmacological or genetic inhibition of IL-6, JAK1/2, and ERK (Cenci et al., 2001; Dubourdeau et al., 2006; Espinosa et al., 2017; Taylor et al., 2016). Finally, IL-6 is an appealing therapeutic target for restoration of inflammatory immunopathology induced by cytokine storm in COVID 19 (Moore and June, 2020). Importantly, superinfections by bacterial and fungal pathogens are increasingly encountered in patients recovering from COVID-19 (Bartoletti et al., 2020). Therefore, our findings provide novel mechanistic view on the emergence of secondary infections caused by opportunistic pathogens in patients with receiving immunomodulatory therapies with inhibitors of IL-6 and JAK/STAT signaling pathways.

Furthermore, our findings lead to a model of phagosome biogenesis that explains the synergistic mechanism of immunosuppressive action of corticosteroid therapy in patients receiving SMKIs, as a result of dual inhibition of two major pathways regulating LAP, PRRs and cytokine signaling (Kyrmizi et al., 2013). Understanding genetic and epigenetic factors regulating LAP responses could have important implications on risk stratifications for susceptibility to infection of these patients. Overall, our work provides the scientific rationale for the development of personalized cytokine-based immunotherapies for sepsis and/or other conditions of immune deregulation, aiming at the restoration of LAP-dependent effector functions and anti-inflammatory pathways in phagocytes. 


\section{STAR Methods}

Key resources table

\section{$\begin{array}{ll}\text { REAGENT } & \text { or } \\ \text { RESOURCE } & \end{array}$}

\section{Antibodies}

Monoclonal Anti-

beta-Tubulin

antibody

produced

Sigma-

in Aldrich

Cat\# T4026; RRID: AB_477577

mouse

Mouse

monoclonal Anti-
Actin Antibody,

Cat\# MAB1501; RRID: AB_2223041

clone $\mathrm{C} 4$

Mouse

monoclonal Anti-

LC3

(microtubule- Nanotools Cat\# 0231-100/LC3-5F10; RRID: AB_2722733

associated protein

1 light chain 3B)

antibody

Rabbit Anti-LC3

polyclonal,

Unconjugated

Novus

Cat\# NB 100-2220; RRID: AB_578334

antibody

Rabbit polyclonal

LC3B Antibody

Novus Cat\# NB600-1384; RRID: AB_669581

Rabbit mAb IL-6 Cell

(D5W4V) XP® Signaling

Cat\#12912; RRID: AB_2798059

Mouse Anti-

CD107a

monoclonal $\quad B D$

Antibody, Biosciences

Cat\# 555798; RRID: AB_396132

Unconjugated,

Clone H4A3

Mouse Anti-

CD63

monoclonal BD

Antibody, Biosciences

Cat\# 556019; RRID: AB_396297

Unconjugated,

Clone H5C6

Mouse Santa Cruz Cat\#sc-17845; RRID: AB_627986 


\section{REAGENT or SOURCE IDENTIFIER}

monoclonal p47- Biotechnolog

phox (D-10) y

antibody

Mouse

$\begin{array}{lll}\text { monoclonal } & \text { BD } & \text { Cat\# 610354; RRID: AB_397744 } \\ \text { p47phox } & \text { Biosciences }\end{array}$

antibody

Rabbit polyclonal Santa Cruz

p22-phox (FL- Biotechnolog Cat\#sc-20781; RRID: AB_2090309

195) antibody $\quad \mathrm{y}$

Mouse

monoclonal Santa Cruz

LAMP-1

antibody

(E-5)

Rabbit polyclonal Santa Cruz

Rab 5B (A-20) Biotechnolog Cat\# sc-598; RRID: AB_2175453

antibody $\quad \mathrm{y}$

Mouse

monoclonal Santa Cruz

Cathepsin D (D- Biotechnolog Cat\# sc-377299

7)

Rabbit Calnexin Novus Cat\# NB100-1974; RRID: AB_10001873
antibody

Rabbit Anti-

ATP6V1B2

Polyclonal

Abcam Cat\# ab73404; RRID: AB_1924799

Antibody,

Unconjugated

p44/42 MAPK

(Erk1/2) (137F5) Cell

Rabbit mAb Signaling

Cat\# 4695; RRID: AB_390779

antibody

Phospho-p44/42

MAPK (Erk1/2)

(Thr202/Tyr204) Cell

(20G11) Rabbit Signaling

Cat\# 4376; RRID: AB_331772

$\mathrm{mAb}$ antibody

Jak2 (D2E12) XP

Rabbit mAb Cell

antibody

Signaling

Cat\# 9367; RRID: AB_1904103

Rabbit polyclonal Minotech N/A anti-GFP 


\section{REAGENT
RESOURCE}

Rabbit polyclonal

$\begin{array}{lll}\text { anti- } & \text { Boussetta } & \text { N/A } \\ \text { PhosphoSer315- } & \text { et al., } 2010 & \end{array}$

p4 $7^{\text {phox }}$ antibody

Rabbit polyclonal

anti-

PhosphoSer328-

p4 $7^{\text {phox }}$ antibody

Boussetta N/A

Rabbit polyclonal

anti-

Dang et al., N/A

PhosphoSer345- 2006

$\mathrm{p} 47^{\text {phox }}$ antibody

$\begin{array}{lcl}\text { Ninein } & \text { (F-5) } & \text { Santa Cruz } \\ \text { Biotechnolog Cat\# sc-376420, RRID:AB_11151570 } & \\ \text { antibody } & y\end{array}$

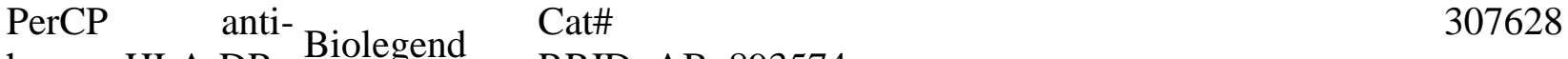

human HLA-DR Biolegend RRID: AB_893574

$\begin{array}{ll}\text { APC anti-human } & \text { Cat\# } \\ \text { CD14 } & \text { RRID: AB_830680 }\end{array}$

Deposited data

Original Wester

Blot and FACS This study gating

https://data.mendeley.com/datasets/cgzsvpmpzc/draft?a=f204d5 e0-349e-4781-914c-96fd2a0f74d8

RNA-seq data This study GEO database (GEO access number: GSE174183)

Chemicals, peptides, and recombinant proteins

\begin{tabular}{|c|c|c|}
\hline Ruxolitinib & $\begin{array}{l}\text { CAYMAN } \\
\text { CHEMICAL } \\
\text { COMPANY }\end{array}$ & 11609; CAS: 941678-49-5 \\
\hline $\begin{array}{l}\text { Recombinant } \\
\text { Murine IL-6 }\end{array}$ & $\begin{array}{l}\text { PEPROTEC } \\
\mathrm{H}\end{array}$ & $216-16$ \\
\hline $\begin{array}{l}2^{\prime}, 7^{\prime}- \\
\text { Dichlorofluoresci } \\
\text { n diacetate }\end{array}$ & $\begin{array}{l}\text { Sigma- } \\
\text { Aldrich }\end{array}$ & D6883; CAS: 4091-99-0 \\
\hline U0126 & Calbiochem & 662005; CAS: 109511-58-2 \\
\hline Propidium Iodide & $\begin{array}{l}\text { Sigma- } \\
\text { Aldrich }\end{array}$ & P4170; CAS: 25535-16-4 \\
\hline $\begin{array}{l}\text { Fluorescein } \\
\text { isothiocyanate- } \\
\text { dextran }\end{array}$ & $\begin{array}{l}\text { Sigma- } \\
\text { Aldrich }\end{array}$ & 46945; CAS: 60842-46-8 \\
\hline
\end{tabular}

Alexa Fluor ${ }^{\mathrm{TM}}$ ThermoFishe

633 NHS Ester r Scientific

A20005 


\section{REAGENT
RESOURCE}

(Succinimidyl

Ester)

Nocodazole

Sigma-

Aldrich

M1404; CAS: 31430-18-9

Paxene

(paclitaxel

IVAX N/A

$6 \mathrm{mg} / \mathrm{ml}$ )

S3I-201

Sigma-

Aldrich

SML0330; CAS: 501919-59-1

Critical commercial assays

Mouse IL-6

ELISA MAX BioLegend Cat:430304

Deluxe kit

Human IL-

6 Uncoated Elisa Invitrogen REF:88-7066-22

kit

Experimental models: Cell lines

NCTC clone 929

[L cell, L-929,

derivative of ATCC CCL-1

Strain L]

Experimental models: Organisms/strains

Aspergillus

fumigatus

ATCC ATCC 46645

Mouse: $I L 6^{+/+}$of

$129 \times \mathrm{C} 57 \mathrm{BL} / 6$

genetic

(Venihaki

et al., 2001) N/A

background

Mouse: $I L 6^{-/}$of

$129 \times \mathrm{C} 57 \mathrm{BL} / 6$

genetic

(Venihaki

et al., 2001) N/A

background

Mouse: C57BL/6 The Jackson

(B6)

Laboratory

000664

RIKEN

Mouse: GFP-LC3 BioResource RBRC00806

Center

\section{Software and algorithms}

GraphPad Prism GraphPad 5

Software

https://www.graphpad.com/ 


\begin{tabular}{|c|c|c|}
\hline $\begin{array}{l}\text { REAGENT } \\
\text { RESOURCE }\end{array}$ & or SOURCE & IDENTIFIER \\
\hline Endnote $x 9$ & $\begin{array}{l}\text { Endnote } \\
\text { Software }\end{array}$ & https://endnote.com/ \\
\hline ImageJ & $\begin{array}{l}\text { Image } \\
\text { Software }\end{array}$ & $\mathrm{J}_{\text {https://imagej-nih-gov.proxy.insermbiblio.inist.fr/ij/ }}$ \\
\hline
\end{tabular}

Other

Graphical

Abstract

BioRender https://app.biorender.com

\section{Resource availability}

\section{Lead contact}

Further information and requests for resources and reagents should be directed to and will be fulfilled by the Lead Contact, Georgios Chamilos (hamilos@imbb.forth.gr).

\section{Materials availability}

All the data that support the findings of this study will be made available upon request made to the Lead Contact.

\section{Data and code availability}

The published article includes all datasets generated or analyzed during this study. The RNAseq data have been deposited in the GEO database (GEO access number: GSE174183). Original FACS analysis and western blots data were deposited at Mendeley at: https://data.mendeley.com/datasets/cgzsvpmpzc/draft?a=f204d5e0-349e-4781-914c-

96fd2a0f74d8

\section{Experimental model and subject details}

\section{Human study subjects and clinical specimens}

All consecutive adult patients admitted to the ICU of a tertiary center Hospital (University Hospital of Crete) from April 2014 to December 2017 with the clinical diagnosis of community acquired septic shock, and a high index of disease severity (APACHE II score $\geq 8$ ) were eligible for the study. Additional sepsis patients were enrolled between December 2019 and April 2020. The diagnosis of septic shock was based on Standardized Criteria (Singer et al., 2016) as follows: an identifiable site of infection and at least two of the signs of systemic inflammatory response syndrome (SIRS); and 2) arterial blood pressure of $<90 \mathrm{mmHg}$ despite adequate fluid resuscitation and requiring vasopressor therapy. Additional patients with septic shock admitted to the medical ICU of Cochin Hospital (Paris, France) were enrolled in 2017.

Exclusion criteria were an age less than 18 years, pregnancy, do-not-resuscitate orders on admission, hematologic malignancy, immunodeficiency defined as HIV infection, presence of 
bone marrow or solid organ transplantation, daily corticosteroid therapy greater than $0.5 \mathrm{mg} / \mathrm{kg}$ prednisone equivalent, cytokine-blocking agents, chemotherapy or any other immunosuppressive treatments. We enrolled healthy volunteers as controls for functional studies.

Approval for the collection of clinical information and tissue samples from septic patients and control healthy individuals was obtained from the Ethics Committee of the University Hospital of Heraklion, Crete, Greece (5159/2014 and 10925/2018) from the appropriate Ethics Committee in France (CPP Ile-de France 3, ref \#S.C. 3351). The patient or next of kin and each healthy volunteer provided written informed consent in accordance with the Declaration of Helsinki. Blood was collected on the day of sepsis diagnosis (Day 1) and later on following sepsis recovery (Day 7).

\section{Data collection}

\section{Baseline data}

Baseline data collection included demographics (age and physician-assigned gender), comorbidities, and immunosuppressive medications.

\section{Characteristics of the primary infection}

The primary site of infection and microbiological data on the pathogens isolated were recorded. We also recorded the APACHE II score, and the SOFA scores on the day of admission in the ICU and at Day 7.

\section{Secondary infections}

Secondary infection in septic shock patients was defined as a new infection acquired more than 48 hours after admission to the ICU. The diagnosis of a secondary infection was performed according to the criteria of the Centers for Disease Control and Prevention (CDC, 2008) (Horan et al., 2008). Infection was differentially diagnosed from colonization according to the CDC criteria and required one or more new antibiotics. The time, site and pathogen isolated for the secondary infection were recorded, and the analysis was restricted to the first episode of secondary infection at the same site.

\section{Endpoints}

Mortality, as well as hospital and ICU length of stay were collected.

\section{Functional studies}

Blood was immediately processed upon collection, $\mathrm{CD}_{1} 4^{+}$monocytes were isolated, stimulated with Aspergillus conidia and fixed on coverslips for immunostaining and analysis. Total RNA from 1 million unstimulated $\mathrm{CD}_{1} 4^{+}$monocytes lysed in Trizol reagent (Invitrogen) was kept in $80^{\circ} \mathrm{C}$. Evaluation of $\mathrm{LC}^{+}$phagosomes and other markers of phagosome biogenesis was performed in batches of paired samples of each individual patient (Day 1, Day 7) as compared to 
the LAPosome formation in monocytes obtained from healthy volunteers (control) from the blood bank of the Hospital.

\section{Mouse models}

C57BL/6 mice (obtained from the IMBB Institute, Crete), IL6 ${ }^{-/-}$and IL6 ${ }^{+/+}$mice of $129 \times \mathrm{C} 57 \mathrm{BL} / 6$ genetic background (Venihaki et al., 2001), GFP-LC3 mice (RIKEN BioResource Center), Atg5 conditional KO mice generated by crossing Atg $5^{f l o x f f l o x}$ mice (RIKEN BioResource Center; (Akoumianaki et al., 2016)) and VavCre ${ }^{+/+}$mice (Akoumianaki et al., 2016), were maintained in grouped cages in a High-Efficiency Particulate Air-filtered environmentally controlled virus-free facility $\left(24^{\circ} \mathrm{C}, 12 / 12 \mathrm{~h}\right.$ light/dark cycle), and fed by standard chow diet and water ad libitum. All experiments were approved by the local ethics committee of the University of Crete Medical School, Greece in line with the corresponding national and European Union legislation (animal protocols 07/05/14-6167 and 17/07/2017-147075).

\section{Polymicrobial sepsis Model in mice}

Pathogen-free, male C57BL/6, 8-10 weeks old (25-30 gr) were subjected to cecal ligation and puncture (CLP) polymicrobial sepsis model (Benjamim et al., 2003, 2005). In brief, mice were anesthetized prior to surgery, by intraperitoneal injection of a ketamine/xylazine mixture. Under sterile conditions, a longitudinal skin midline incision was made and the cecum was exposed to the surface. The cecum was partially ligated (60\% of the cecum in reference to the distal pole) with a 4-0 silk suture without disrupting the intestinal continuity. The ligated cecum was punctured through and through with a 21-gauge needle three times (six punctures in total) in order to achieve severe sepsis conditions. For mild sepsis, only one puncture was performed. Sham-operated mice underwent the same surgery but without cecal ligation and puncture and were used as controls. Six hours after induction of polymicrobial sepsis, sham or CLP operated mice were started on antibiotic treatment with $10 \mathrm{mg} / \mathrm{kg}$ Imipenem/cilastatin. The antibiotic was administered every 12 hours for a period of three days. On Day 7 following recovery from sepsis, sham and CLP mice were challenged by intratracheal installation of $5 \times 10^{7}$ A. fumigatus conidia. After 48 hours of infection, mice were euthanized, their lungs were harvested and homogenized in phosphate buffer saline. The fungal burden was evaluated by CFU plating of lung homogenates.

\section{Microorganisms and culture conditions}

All A. fumigatus strains used ( $\triangle p k s P$ and the isogenic wild type strain ATCC46645) have been described previously (Akoumianaki et al., 2016). All strains were grown on YAG agar plates for 3 days at $37^{\circ} \mathrm{C}$. Fungal conidia (spores) were harvested by gentle shaking in the presence of sterile $0.1 \%$ Tween 20 in PBS, washed twice with PBS, filtered through a $40 \mu \mathrm{m}$-pore-size cell strainer (Falcon) to separate conidia from contaminating mycelium, counted by a hemocytometer, and suspended at a concentration of $10^{8}$ spores $/ \mathrm{mL}$. When indicated, the conidia were labeled with FITC or Alexa Fluor 633 succinimidyl ester dye (Invitrogen) as previously described (Akoumianaki et al., 2016). Briefly, freshly harvested conidia $\left(5 \times 10^{7} / 2 \mathrm{~mL}\right.$ in $100 \mathrm{mM} \mathrm{NaHCO}_{3}$, $\mathrm{pH}$ 8.3) were incubated with FITC (Sigma Aldrich, $0.1 \mathrm{mg} / \mathrm{mL}$ final concentration) or Alexa Fluor 633 succinimidyl ester dye (Invitrogen, $1.7 \mathrm{mg} / \mathrm{mL}$ ) at $37^{\circ} \mathrm{C}$ for $2 \mathrm{~h}$ and washed by centrifugation three times in PBS-0.1\% Tween 20. Inactivation of fungal conidia was done by 
exposure to $4 \% \mathrm{FA}$ ( $2 \mathrm{~h}$, room temperature) following by treatment with glycine (100 $\mathrm{mM}$ in PBS) and three washes in PBS and verified by CFU plating. Importantly, we have previously validated that FA-inactivation of $A$. fumigatus conidia does not affect LAPosome formation and PRR signaling activation (Akoumianaki et al., 2016).

A single colony of Pseudomonas aeruginosa clinical isolate (ATCC 27853) isolated from LB plates was grown at a mid-logarithmic phase in LB liquid media the day of infection. Bacterial cells were washed with PBS, adjusted to 0.5 MacFarlane $\left(10^{8}\right.$ bacteria/ml $)$ and immediately used for infection experiments.

\section{Method details}

\section{Isolation and stimulation of primary human monocytes}

Healthy volunteers without any known infectious or inflammatory disorders donated blood. Monocytes from sepsis patients or healthy controls were isolated from PBMCs using magnetic bead separation with anti-CD14 coated beads (MACS Miltenyi Germany) according to the protocol supplemented by the manufacturer. The monocytes were resuspended in RPMI 1640 culture medium supplemented with Hepes $10 \mathrm{mM}$, penicillin-streptomycin $1 \%$, l-glutamine $1 \%$, sodium pyruvate $1 \%$, glucose $25 \mathrm{mM}$ and fetal bovine serum $10 \%$. The cells were counted, and their number was adjusted to $2 \times 10^{6} / \mathrm{mL}$. A total of $2 \times 10^{5}$ monocytes per condition in a final volume of $100 \mu \mathrm{L}$ were allowed to adhere to sterile polylysine coated glass coverslips $(\varnothing 13 \mathrm{~mm})$ for $1 \mathrm{~h}$ followed by stimulation with A. fumigatus conidia at a multiplicity of infection (MOI) of $3: 1$ at $37^{\circ} \mathrm{C}$ for the indicated time point. After stimulation, cells were washed twice with PBS to remove medium and non-phagocytosed spores and cells were fixed for 15 min in $4 \%$ paraformaldehyde or $4 \%$ formaldehyde (w/o MeOH). Subsequently, the coverslips were washed with PBS followed by an extra step of fixation with ice-cold methanol for 10 min at $-20^{\circ} \mathrm{C}$ in case of immunostaining for LC3, after which were stored in PBS at $4{ }^{\circ} \mathrm{C}$ until immunofluorescence staining.

\section{Immunofluorescence staining}

For immunofluorescence imaging, fixed cells were blocked for 15 minutes with $2 \%$ BSA, $0.1 \%$ saponin in PBS, incubated for $1 \mathrm{~h}$ with the indicated primary antibody, washed twice in PBS, stained by the appropriate secondary AlexaFluor secondary Ab (Molecular Probes), followed by DNA staining with $1 \mu \mathrm{M}$ TOPRO-3 iodide (642/661; Invitrogen). For ERK staining coverslips were incubated overnight with the primary antibody at $4{ }^{\circ} \mathrm{C}$. After the washing steps, slides were mounted in Prolong Gold antifade media (Molecular Probes). Images were acquired using a laser-scanning spectral confocal microscope (TCS SP8; Leica), LCS Lite software (Leica), and a 40× Apochromat 1.25 NA oil objective using identical gain settings. A low fluorescence immersion oil (11513859; Leica) was used, and imaging was performed at room temperature. Serial confocal sections at $0.5 \mu \mathrm{m}$ steps within a z-stack spanning a total thickness of 10 to $12 \mu \mathrm{m}$ of the cell were taken, and 3D images were generated using the LCS Lite software to assess for internalized conidia contained within phagosomes. Unless otherwise stated, mean projections of image stacks were obtained using the LCS Lite software. Phagosomes surrounded by a rim of fluorescence of the indicated protein-marker were scored as positive, according to established protocols in our lab. At least 200 phagosomes were analyzed for each experimental condition in 
three independent experiments, in a double blinded fashion by the same investigator (T.A.). Assessment of phagolysosomal fusion was performed in BMDMs pre-loaded with FITC-Dextran. Briefly, BMDMs were pre-loaded with FITC-Dextran for $4 \mathrm{~h}$, media was replaced for $2 \mathrm{~h}$ to allow for labeling of lysosomes, and phagolysosomal fusion was assessed following fungal infection based on the accumulation of FITC-Dextran at the phagosomes.

In addition, phagocytosis rate and phagocytosis index were assessed in primary human monocytes at $30 \mathrm{~min}$ of infection with FITC-labeled A. fumigatus (MOI 3:1). The phagocytic index was expressed with the following formula: (total number of engulfed cells/total number of counted macrophages $) \times$ (number of macrophages containing engulfed cells/total number of counted macrophages $) \times 100$.

\section{Live imaging}

BMDMs were loaded with FITC-Dextran (5 $\mu \mathrm{M}$ final concentration, Invitrogen) for $4 \mathrm{~h}$ washed with PBS cells counted and seeded in chambers for live imaging (627870 Greiner) in HBSS (w/o red phenol, $2 \mathrm{mM} \mathrm{CaCl}, 1 \mathrm{mM} \mathrm{MgCl}_{2}, 20 \mathrm{mM}$ Hepes). After adhesion of the cells, infections with the indicated $A$. fumigatus strain were performed just before live imaging. The acquisition was performed in SP8 Leica converted microscope with 40× Apochromat 1.25 NA water objective using identical imaging setting, and high speed live imaging with the use of a resonant scanner.

\section{Measurement of ROS production}

ROS measurements were performed by means of a dichlorofluorescein assay (Akoumianaki et al., 2016). The Stock solution of dichlorofluorescein diacetate (DCFH-DA) was dissolved in dimethyl sulfoxide (DMSO) to a final concentration of $10 \mathrm{mM}$. Human monocytes $\left(2 \times 10^{5} /\right.$ well) or mouse BMDMs $\left(1 \times 10^{5} /\right.$ well $)$ were plated on 96 -well round bottom plates, incubated at $37^{\circ} \mathrm{C}$ for $30 \mathrm{~min}$ and accordingly stimulated for $1 \mathrm{~h}$ with A. fumigatus conidia (MOI 10:1) or zymosan $(50 \mu \mathrm{g} / \mathrm{ml})$ in the presence of DCFH-DA added to a final concentration of $10 \mu \mathrm{M}$ during the last $30 \mathrm{~min}$. After $30 \mathrm{~min}$ of exposure, the well content was transferred to vials and the fluorescence of the cells was measured by flow cytometry. Cells were acquired on a FACSCalibur (BD Biosciences) and analyzed using FlowJo software (Tree Star).

\section{Generation of murine BMDMS}

BMDMs were generated by culturing bone marrow cells obtained from 8 to 12 weeks old female mice in DMEM, supplemented with L929 cell-conditioned medium (30\%). The resulting cultures consisted of macrophages ( $>95 \%$ purity), as determined by staining for F4/80 and flow cytometry.

\section{Isolation of splenic monocytes}

Mouse monocytes were isolated from single cell suspension of spleens obtained from control and septic mice. Briefly, splenic cells were incubated for 30 min with a Ly6C biotinylated antibody and monocytes were isolated with magnetic streptavidin beads. 


\section{Killing of A. fumigatus by monocytes/macrophages}

Primary human monocytes $\left(2 \times 10^{5} /\right.$ well $)$ or BMDMs $\left(1 \times 10^{5} /\right.$ well $)$ were plated onto 96-well round bottom plates for $1 \mathrm{~h}$, and subsequently infected with the indicated A. fumigatus strain at an MOI of 1:10 (conidia: target cell ratio) for $1 \mathrm{~h}$ at $37^{\circ} \mathrm{C}$ with or without the indicated concentration of JAK2 inhibitor (ruxolitinib) or STAT3 inhibitor (S3I-201) added 30 min before infection. Medium containing non-adherent, non-phagocytosed conidia was removed, wells were washed three times using warm PBS, and new media with or without the presence of the corresponding inhibitor were added. Monocytes were then allowed to kill conidia for $6 \mathrm{~h}$ before intracellular conidia were harvested by lysis of monocytes with $0.5 \%$ Triton-X. The process of cellular lysis was confirmed by light microscopy and the killing of A. fumigatus conidia was assessed by CFU plating. Each condition was performed in quadruplicate with monocytes obtained from 4 different donors. In other experiments killing of FITC-labeled conidia by BMDMs was assessed by viability staining with Propidium Iodide (PI). Briefly, murine BMDMs were allowed to ingest FITC-labeled A. fumigatus conidia at an MOI of $1: 1$ for $1 \mathrm{~h} \mathrm{at} 37^{\circ} \mathrm{C}$ in 24 well plates. BMDMs were allowed to kill conidia for $6 \mathrm{~h}$ before intracellular conidia were harvested by sonication (Akoumianaki et al., 2016). Next, cell lysates were stained with Propidium Iodide (PI) to assess the viability of A. fumigatus conidia. FITC labeled PFA-killed or live A. fumigatus conidia served as controls for PI staining.

\section{Killing of Pseudomonas by BMDMs}

BMDMs $\left(2 \times 10^{5}\right.$ per condition) from IL- $6^{-/-}(\mathrm{KO})$ or control IL- $6^{+/+}$mice were seeded overnight in 96 well flat bottom plates and infected with Pseudomonas aeruginosa (ATCC 27853 grown at a mid-logarithmic phase) in antibiotic free media at a MOI of 10:1 (target: effector ratio) with or without supplementation of culture media with IL-6 at a final concentration of $10 \mathrm{ng} / \mathrm{ml}$. Cells were extensively washed at $1 \mathrm{~h}$ of infection with warm PBS and a cell-impermeable antibiotic (gentamicin $300 \mu \mathrm{g} / \mathrm{ml}$ ) was added in culture media for $30 \mathrm{~min}$ to kill remaining extracellular bacteria. The amount of phagocytosed bacteria at $1 \mathrm{~h}$ of infection was assessed following cell lysis by CFU plating. Bacterial killing by BMDMs was assessed by CFU counts of intracellular bacteria at $12 \mathrm{~h}$ of infection.

\section{Western blot analysis}

Primary human monocytes or murine BMDMs were stimulated with Aspergillus fumigatus conidia or PMA for the indicated time points at MOI 10:1 with or without the presence of the indicated inhibitor. Cells were washed once in PBS prior to lysis in 1\% NP-40 containing RIPA buffer (50 mM Tris [pH 7.4], $150 \mathrm{mM} \mathrm{NaCl}, 1 \mathrm{mM}$ EDTA, 0,25\% sodium deoxycholate, $1 \mathrm{mM}$ $\mathrm{NaF}, 1 \mathrm{mM} \mathrm{Na} \mathrm{VO}_{4}, 1 \mathrm{mM}$ PMSF plus a mixture of protease inhibitors [Roche Molecular Biochemicals]). For analysis of p47phox phosphorylation, a mixture of phosphatase inhibitors (PhosSTOP; Roche Molecular Biochemicals) was added to the lysis buffer. Cell lysis was performed on ice for 30 minutes, and samples were centrifuged. After protein estimation of supernatants, addition of SDS sample buffer and boiling, samples were separated on SDS-PAGE and transferred to polyvinylidene difluoride (PVDF) membranes. Western blotting was performed according to the instructions of the manufacturer using the indicated primary antibodies: Secondary antibodies used in western blotting were purchased from Cell Signaling 
(anti-rabbit HRP, anti-goat HRP) as well as Millipore (anti-mouse HRP). The blots were developed using chemiluminescence (ECL; Thermo Scientific).

\section{Measurement of cytokines}

Supernatants of stimulated human monocytes were collected after overnight culture and were stored at $-20^{\circ} \mathrm{C}$ until analysis. Cytokine levels in the supernatants were determined by using ELISA kits for human IL-1 $\beta$, IL-1Ra, and TNF (R\&D Systems) and IL-6 (Sanquin) according to the manufacturer's instructions.

\section{RNA extraction}

Monocytes isolated from different groups of sepsis patients stratified by LAP responses were lysed in Trizol reagent and stored at $-80^{\circ} \mathrm{C}$. Total RNA was extracted from cells using the QIAGEN RNeasy RNA extraction kit, using on-column DNaseI treatment. Ribosomal RNA was removed using the riboZero rRNA removal kit.

\section{Library preparation for RNA-seq}

RNA samples were shipped to the sequencing facility. RNA integrity was measured using Agilent TapeStation System. All samples had good qualities (RIN score >=8). RNA libraries were prepared to enrich for polyA tail-RNA using Bioscientific NEXTflex-Rapid-Directional mRNA-seq kits. RNA libraries were prepared with the Caliper SciClone system. The libraries were sequenced using the NextSeq500 High Output kit V2 (Illumina) for 75 cycles.

\section{RNA-seq data analysis}

Raw RNA-seq reads were aligned to the human genome version 37 using STAR (v2.5.1b) (Dobin et al., 2013) HTSeq-count package (v0.61) was used to count and normalize the number of reads mapped to each gene (Anders et al., 2015). Differential expression was determined using DEseq2 (v.1.12.4) (Love et al., 2014). For subsets of genes involved in autophagy, LAP, IL-6 signaling and ERK pathways (refer to Figure S7), normalized counts were log transformed and comparisons between patient groups was performed using One-way ANOVA and corrected for the number of genes tested (Bonferroni) (FDR $<=0.05$ and fold change $>1.5$ ). The RNAseq data have been deposited in the GEO database (GEO access number: GSE174183).

\section{Quantification and statistical analysis}

For in vivo results, each $\mathrm{n}$ value represents an individual experiment and mouse. For the human in vitro experiments, each $\mathrm{n}$ refers to a separate independent experiment. The number of experiments for each figure can be found in the figure legends. The data were expressed as mean \pm SEM. Data were tested for Gaussian distribution using a Shapiro-Wilk normality analysis. For normally distributed data two-sided unpaired Student's $t$ test was used and for non-Gaussian data two sided unpaired Mann-Whitney tests were used to compute statistical significance between two groups. One-way ANOVA with the indicated post hoc test was used for multiple comparisons $(P<0.05$ was considered statistically significant). Survival analysis and studies on 
the cumulative probability of infection in patients over time were performed by log-rank (Mantel-Cox) test. Analysis was performed using the GraphPad Prism software (version VII). All in vitro experiments were performed at least in triplicate and replicated at least twice.

\section{Acknowledgments}

The authors would like to thank Yiannis Talianidis and Agostinho Carvalho for helpful suggestions. T.A.'s work was supported by a grant from Hellenic Foundation for Research and innovation (HFRI) (\#1787); G.C. was supported by grants from the Greek State Scholarship Foundation (I.K.Y.), the Hellenic General Secretariat for Research and Technology-Excellence program (ARISTEIA), an Advanced Research Grant from Institut Mérieux (\#4719), H2020-SC1BHC-2018-2020 (HDM-FUN 847507), and an ERC Consolidator Grant (iMAC-FUN-\#864947); J.-P.L. was supported by European Community's Seventh Framework Programme (FP7/20072013) under grant agreement 260338 ALLFUN and ANR-10-BLAN-1309 hydrophobin, and the Association Vaincre La Mucoviscidose (RF20140501052/1/1/141). M.G.N. is supported by an ERC Consolidator grant (\#310372) and a Spinoza grant of the Netherlands Organization for Scientific Research (NWO). This study was supported by a TOP grant (project number: 91214016) of the Netherlands Organization for Health Research and Development (ZonMw) (to M.G.N.).

\section{Author contributions}

T.A. performed and analyzed most of the experiments in human patients and mice, designed and established protocols on the in vivo model of sepsis, live imaging studies, and assays for evaluation of microtubule organization, and participated in the writing of the manuscript. R.B. and M.G. performed experiments on human samples. M.G. performed analysis of cytokine from sepsis patients. D.G., K.V., F.P., and E.D. collected and analyzed clinical data and provided samples from patients with sepsis; M.V. provided the IL- $6^{-/-}$mice and analyzed data; J.E.-B. provided reagents and analyzed data; K.T.T.L. and V.K. performed RNA-seq analysis; F.L.V.d.V. and M.G.N. were involved in the design of experiments on human studies, analyzed data, and participated in the writing of the manuscript; E.D. performed histopathological studies. J.-P.L. analyzed data and provided discussions and suggestions throughout the study; G.C. conceived and supervised the study, was involved in the design and evaluation of all of the experiments, and wrote the manuscript along with comments from co-authors.

\section{Declaration of interests}

The authors declare no competing interests.

\section{ACKNOWLEDGMENTS}

The authors would like to thank Yiannis Talianidis and Agostinho Carvalho for helpful suggestions. T.A.'s work was supported by a grant from Hellenic Foun- dation for Research and innovation (HFRI) (\#1787); G.C. was supported by grants from the Greek State Scholarship Foundation (I.K.Y.), the Hellenic Gen- eral Secretariat for Research and Technology-Excellence 
program (ARIS- TEIA), an Advanced Research Grant from Institut Mé rieux (\#4719), H2020SC1-BHC-2018-2020 (HDM-FUN 847507), and an ERC Consolidator Grant (iMAC-FUN\#864947); J.-P.L. was supported by European Community's Sev- enth Framework Programme (FP7/2007-2013) under grant agreement 260338 ALLFUN and ANR-10-BLAN-1309 hydrophobin, and the Association Vaincre La Mucoviscidose (RF20140501052/1/1/141). M.G.N. is supported by an ERC Consolidator grant (\#310372) and a Spinoza grant of the Netherlands Organization for Scientific Research (NWO). This study was supported by a TOP grant (project number: 91214016) of the Netherlands Organization for Health Research and Development (ZonMw) (to M.G.N.).

\section{AUTHOR CONTRIBUTIONS}

T.A. performed and analyzed most of the experiments in human patients and mice, designed and established protocols on the in vivo model of sepsis, live imaging studies, and assays for evaluation of microtubule organization, and participated in the writing of the manuscript. R.B. and M.G. performed exper- iments on human samples. M.G. performed analysis of cytokine from sepsis patients. D.G., K.V., F.P., and E.D. collected and analyzed clinical data and provided samples from patients with sepsis; M.V. provided the IL-6- - mice and analyzed data; J.E.-B. provided reagents and analyzed data; K.T.T.L. and V.K. performed RNA-seq analysis; F.L.v.d.V. and M.G.N. were involved in the design of experiments on human studies, analyzed data, and partici- pated in the writing of the manuscript; E.D. performed histopathological studies. J.-P.L. analyzed data and provided discussions and suggestions throughout the study; G.C. conceived and supervised the study, was involved in the design and evaluation of all of the experiments, and wrote the manu- script along with comments from co-authors.

\section{DECLARATION OF INTERESTS}

The authors declare no competing interests. 


\section{REFERENCES}

Akoumianaki, T., Kyrmizi, I., Valsecchi, I., Gresnigt, M.S., Samonis, G., Drakos, E., Boumpas, D., Muszkieta, L., Prevost, M.-C., Kontoyiannis, D.P., et al. (2016). Aspergillus cell wall melanin blocks LC3-associated phagocytosis to promote pathogenicity. Cell Host Microbe 19, 79-90.

Anders, S., Pyl, P.T., and Huber, W. (2015). HTSeq-a Python framework to work with highthroughput sequencing data. Bioinformatics 31, 166-169.

Arnold, C. (2018). News feature: the quest to solve sepsis. Proc. Natl. Acad. Sci. USA 115, 3988-3991.

Bacher, P., Heinrich, F., Stervbo, U., Nienen, M., Vahldieck, M., Iwert, C., Vogt, K., Kollet, J., Babel, N., Sawitzki, B., et al. (2016). Regulatory T cell specificity directs tolerance versus allergy against aeroantigens in humans. Cell 167, 1067-1078.e16.

Bae, S., Hwang, H.J., Kim, M.Y., Kim, M.J., Chong, Y.P., Lee, S.O., Choi, S.H., Kim, Y.S., Woo, J.H., and Kim, S.H. (2020). Invasive pulmonary aspergillosis in patients with severe fever With thrombocytopenia syndrome. Clin. Infect. Dis. 70, 1491-1494.

Bambouskova, M., Gorvel, L., Lampropoulou, V., Sergushichev, A., Loginicheva, E., Johnson, K., Korenfeld, D., Mathyer, M.E., Kim, H., Huang, L.-H., et al. (2018). Electrophilic properties of itaconate and derivatives regu- late the IkBz -ATF3 inflammatory axis. Nature 556, 501-504.

Bartoletti, M., Pascale, R., Cricca, M., Rinaldi, M., Maccaro, A., Bussini, L., Fornaro, G., Tonetti, T., Pizzilli, G., Francalanci, E., et al. (2020). Epidemiology of invasive pulmonary aspergillosis among COVID-19 intubated patients: a prospective study. Clin. Infect. Dis.

Bassetti, M., Garnacho-Montero, J., Calandra, T., Kullberg, B., Dimopoulos, G., Azoulay, E., Chakrabarti, A., Kett, D., Leon, C., Ostrosky-Zeichner, L., et al. (2017). Intensive care medicine research agenda on invasive fungal infec- tion in critically ill patients. Intensive Care Med 43, $1225-1238$.

Benjamim, C.F., Hogaboam, C.M., Lukacs, N.W., and Kunkel, S.L. (2003). Septic mice are susceptible to pulmonary aspergillosis. Am. J. Pathol. 163, 2605-2617.

Benjamim, C.F., Lundy, S.K., Lukacs, N.W., Hogaboam, C.M., and Kunkel, S.L. (2005). Reversal of long-term sepsis-induced immunosuppression by dendritic cells. Blood 105, 35883595.

Blocker, A., Severin, F.F., Burkhardt, J.K., Bingham, J.B., Yu, H., Olivo, J.C., Schroer, T.A., Hyman, A.A., and Griffiths, G. (1997). Molecular requirements for bi-directional movement of phagosomes along microtubules. J. Cell Biol. 137, 113-129.

Boomer, J.S., To, K., Chang, K.C., Takasu, O., Osborne, D.F., Walton, A.H., Bricker, T.L., Jarman, S.D., 2nd, Kreisel, D., Krupnick, A.S., et al. (2011). Immunosuppression in patients who die of sepsis and multiple organ failure. JAMA 306, 2594-2605. 
Boussetta, T., Gougerot-Pocidalo, M.-A., Hayem, G., Ciappelloni, S., Raad, H., Arabi Derkawi, R., Bournier, O., Kroviarski, Y., Zhou, X.Z., Malter, J.S., et al. (2010). The prolyl isomerase Pin1 acts as a novel molecular switch for TNF-alpha-induced priming of the NADPH oxidase in human neutrophils. Blood 116, 5795-5802.

Cenci, E., Mencacci, A., Casagrande, A., Mosci, P., Bistoni, F., and Romani, L. (2001). Impaired antifungal effector activity but not inflammatory cell recruit- ment in interleukin-6-deficient mice with invasive pulmonary aspergillosis. J. Infect. Dis. 184, 610-617.

Chamilos, G., Lionakis, M.S., and Kontoyiannis, D.P. (2018a). Call for action: invasive fungal infections associated with ibrutinib and other small molecule ki- nase inhibitors targeting immune signaling pathways. Clin. Infect. Dis. 66, 140-148.

Chamilos, G., Lionakis, M.S., and Kontoyiannis, D.P. (2018b). Reply to Bazaz and denning. Clin. Infect. Dis. 67, 157-159.

Cheng, S.C., Scicluna, B.P., Arts, R.J., Gresnigt, M.S., Lachmandas, E., Giamarellos-Bourboulis, E.J., Kox, M., Manjeri, G.R., Wagenaars, J.A., Cremer, O.L., et al. (2016). Broad defects in the energy metabolism of leuko- cytes underlie immunoparalysis in sepsis. Nat. Immunol. 17, 406413.

Chiswick, E.L., Mella, J.R., Bernardo, J., and Remick, D.G. (2015). Acute- phase deaths from murine polymicrobial sepsis are characterized by innate immune suppression rather than exhaustion. J. Immunol. 195, 3793-3802.

Chu, H., Khosravi, A., Kusumawardhani, I.P., Kwon, A.H., Vasconcelos, A.C., Cunha, L.D., Mayer, A.E., Shen, Y., Wu, W.-L., Kambal, A., et al. (2016). Gene- microbiota interactions contribute to the pathogenesis of inflammatory bowel disease. Science 352, 1116-1120.

Colombo, A.L., de Almeida Ju' nior, J.N., Slavin, M.A., Chen, S.C., and Sorrell, T.C. (2017). Candida and invasive mould diseases in non-neutropenic critically ill patients and patients with haematological cancer. Lancet Infect. Dis. 17, e344-e356.

Dang, P.M., Stensballe, A., Boussetta, T., Raad, H., Dewas, C., Kroviarski, Y., Hayem, G., Jensen, O.N., Gougerot-Pocidalo, M.A., and El-Benna, J. (2006). A specific p47phox -serine phosphorylated by convergent MAPKs mediates neutrophil NADPH oxidase priming at inflammatory sites. J. Clin. Invest. 116, 2033-2043.

de Luca, A., Smeekens, S.P., Casagrande, A., Iannitti, R., Conway, K.L., Gresnigt, M.S., Begun, J., Plantinga, T.S., Joosten, L.A., van der Meer, J.W., et al. (2014). IL-1 receptor blockade restores autophagy and reduces inflam- mation in chronic granulomatous disease in mice and in humans. Proc. Natl. Acad. Sci. USA 111, 3526-3531.

Dimopoulos, G., Piagnerelli, M., Berre' , J., Eddafali, B., Salmon, I., and Vincent, J.L. (2003). Disseminated aspergillosis in intensive care unit patients: an au- topsy study. J. Chemother. 15, $71-75$.

Dioverti, M.V., Abu Saleh, O.M., and Tande, A.J. (2018). Infectious complica- tions in patients on treatment with Ruxolitinib: case report and review of the literature. Infect. Dis. (Lond) 50, 
$381-387$.

Dobin, A., Davis, C.A., Schlesinger, F., Drenkow, J., Zaleski, C., Jha, S., Batut, P., Chaisson, M., and Gingeras, T.R. (2013). STAR: ultrafast universal RNA-seq aligner. Bioinformatics 29, 1521.

Do“cke, W.D., Randow, F., Syrbe, U., Krausch, D., Asadullah, K., Reinke, P., Volk, H.D., and Kox, W. (1997). Monocyte deactivation in septic patients: restoration by IFN-gamma treatment. Nat. Med. 3, 678-681.

Domínguez-Andre' s, J., Novakovic, B., Li, Y., Scicluna, B.P., Gresnigt, M.S., Arts, R.J.W., Oosting, M., Moorlag, S.J.C.F.M., Groh, L.A., Zwaag, J., et al. (2019). The itaconate pathway is a central regulatory node linking innate im- mune tolerance and trained immunity. Cell Metab 29, 211-220.e5.

Dubourdeau, M., Athman, R., Balloy, V., Huerre, M., Chignard, M., Philpott, D.J., Latge' , J.P., and Ibrahim-Granet, O. (2006). Aspergillus fumigatus induces innate immune responses in alveolar macrophages through the MAPK pathway independently of TLR2 and TLR4. J. Immunol. 177, 3994-4001.

El-Benna, J., Dang, P.M., Gougerot-Pocidalo, M.A., Marie, J.C., and Braut- Boucher, F. (2009). p47phox, the phagocyte NADPH oxidase/NOX2 organizer: structure, phosphorylation and implication in diseases. Exp. Mol. Med. 41, 217-225.

Espinosa, V., Dutta, O., McElrath, C., Du, P., Chang, Y.J., Cicciarelli, B., Pitler, A., Whitehead, I., Obar, J.J., Durbin, J.E., et al. (2017). Type III interferon is a critical regulator of innate antifungal immunity. Sci. Immunol. 2, eaan5357.

Favata, M.F., Horiuchi, K.Y., Manos, E.J., Daulerio, A.J., Stradley, D.A., Feeser, W.S., Van Dyk, D.E., Pitts, W.J., Earl, R.A., Hobbs, F., et al. (1998). Identification of a novel inhibitor of mitogen-activated protein kinase kinase. J. Biol. Chem. 273, 18623-18632.

Forbester, J.L., Lees, E.A., Goulding, D., Forrest, S., Yeung, A., Speak, A., Clare, S., Coomber, E.L., Mukhopadhyay, S., Kraiczy, J., et al. (2018). Interleukin-22 promotes phagolysosomal fusion to induce protection against Salmonella enterica Typhimurium in human epithelial cells. Proc. Natl. Acad. Sci. USA 115, 10118-10123.

Harrison, R.E., Bucci, C., Vieira, O.V., Schroer, T.A., and Grinstein, S. (2003). Phagosomes fuse with late endosomes and/or lysosomes by extension of membrane protrusions along microtubules: role of Rab7 and RILP. Mol. Cell. Biol. 23, 6494-6506.

Heckmann, B.L., and Green, D.R. (2019). LC3-associated phagocytosis at a glance. J. Cell Sci. $132, \mathrm{jcs} 222984$.

Heckmann, B.L., Teubner, B.J.W., Tummers, B., Boada-Romero, E., Harris, L., Yang, M., Guy, C.S., Zakharenko, S.S., and Green, D.R. (2019). LC3- Associated Endocytosis Facilitates betaamyloid Clearance and Mitigates Neurodegeneration in Murine Alzheimer's disease. Cell 178, 536-551.e14. 
Hoetzenecker, W., Echtenacher, B., Guenova, E., Hoetzenecker, K., Woelbing, F., Bru€ck, J., Teske, A., Valtcheva, N., Fuchs, K., Kneilling, M., et al. (2011). ROS-induced ATF3 causes susceptibility to secondary infections during sepsis-associated immunosuppression. Nat. Med. 18, $128-134$.

Horan, T.C., Andrus, M., and Dudeck, M.A. (2008). CDC/NHSN surveillance definition of health care-associated infection and criteria for specific types of infections in the acute care setting. Am. J. Infect. Control 36, 309-332.

Hotchkiss, R.S., Monneret, G., and Payen, D. (2013). Sepsis-induced immuno- suppression: from cellular dysfunctions to immunotherapy. Nat. Rev. Immunol. 13, 862-874.

Jay, J., Hammer, A., Nestor-Kalinoski, A., and Diakonova, M. (2015). JAK2 tyrosine kinase phosphorylates and is negatively regulated by centrosomal protein Ninein. Mol. Cell. Biol. 35, 111-131.

Jung, J.Y., and Robinson, C.M. (2014). IL-12 and IL-27 regulate the phagoly- sosomal pathway in mycobacteria-infected human macrophages. Cell Commun. Signal. 12, 16.

Kyrmizi, I., Ferreira, H., Carvalho, A., Figueroa, J.A.L., Zarmpas, P., Cunha, C., Akoumianaki, T., Stylianou, K., Deepe, G.S., Jr., Samonis, G., et al. (2018). Calcium sequestration by fungal melanin inhibits calcium-calmodulin signal- ling to prevent LC3-associated phagocytosis. Nat. Microbiol. 3, 791-803.

Kyrmizi, I., Gresnigt, M.S., Akoumianaki, T., Samonis, G., Sidiropoulos, P., Boumpas, D., Netea, M.G., van de Veerdonk, F.L., Kontoyiannis, D.P., and Chamilos, G. (2013). Corticosteroids block autophagy protein recruitment in Aspergillus fumigatus phagosomes via targeting dectin-1/Syk kinase signaling. J. Immunol. 191, 1287-1299.

Leentjens, J., Kox, M., Koch, R.M., Preijers, F., Joosten, L.A., van der Hoeven, J.G., Netea, M.G., and Pickkers, P. (2012). Reversal of immunoparalysis in hu- mans in vivo: a double-blind, placebo-controlled, randomized pilot study. Am. J. Respir. Crit. Care Med. 186, 838-845.

Love, M.I., Huber, W., and Anders, S. (2014). Moderated estimation of fold change and dispersion for RNA-seq data with DESeq2. Genome Biol 15, 550.

Lussana, F., Cattaneo, M., Rambaldi, A., and Squizzato, A. (2018). Ruxolitinib- associated infections: a systematic review and meta-analysis. Am. J. Hematol. 93, 339-347.

Ma, X., and Sayeski, P.P. (2007). Identification of tubulin as a substrate of Jak2 tyrosine kinase and its role in Jak2-dependent signaling. Biochemistry 46, 7153-7162.

Makni-Maalej, K., Chiandotto, M., Hurtado-Nedelec, M., Bedouhene, S., Gougerot-Pocidalo, M.A., Dang, P.M., and El-Benna, J. (2013). Zymosan induces NADPH oxidase activation in human neutrophils by inducing the phosphorylation of p47phox and the activation of Rac2: involvement of protein tyrosine kinases, PI3kinase, PKC, ERK1/2 and p38MAPkinase. Biochem. Pharmacol. 85, 92-100. 
Martinez, J., Almendinger, J., Oberst, A., Ness, R., Dillon, C.P., Fitzgerald, P., Hengartner, M.O., and Green, D.R. (2011). Microtubule-associated protein 1 light chain 3 alpha (LC3)-associated phagocytosis is required for the efficient clearance of dead cells. Proc. Natl. Acad. Sci. USA 108, 17396-17401.

Martinez, J., Cunha, L.D., Park, S., Yang, M., Lu, Q., Orchard, R., Li, Q.Z., Yan, M., Janke, L., Guy, C., et al. (2016). Noncanonical autophagy inhibits the auto- inflammatory, lupus-like response to dying cells. Nature 533, 115-119.

Martinez, J., Malireddi, R.K., Lu, Q., Cunha, L.D., Pelletier, S., Gingras, S., Orchard, R., Guan, J.-L., Tan, H., Peng, J., et al. (2015). Molecular character- ization of LC3-associated phagocytosis reveals distinct roles for Rubicon, NOX2 and autophagy proteins. Nat. Cell Biol. $17,893-906$.

Meersseman, W., Vandecasteele, S.J., Wilmer, A., Verbeken, E., Peetermans, W.E., and Van Wijngaerden, E. (2004). Invasive aspergillosis in critically ill pa- tients without malignancy. Am. J. Respir. Crit. Care Med. 170, 621-625.

Moore, J.B., and June, C.H. (2020). Cytokine release syndrome in severe COVID-19. Science $368,473-474$.

Morris, E.J., Kawamura, E., Gillespie, J.A., Balgi, A., Kannan, N., Muller, W.J., Roberge, M., and Dedhar, S. (2017). Stat 3 regulates centrosome clustering in cancer cells via stathmin/PLK1. Nat. Commun. 8, 15289. Murray, R.Z., Kay, J.G., Sangermani, D.G., and Stow, J.L. (2005). A role for the phagosome in cytokine secretion. Science 310, 1492-1495.

Ng, D.C., Lin, B.H., Lim, C.P., Huang, G., Zhang, T., Poli, V., and Cao, X. (2006). Stat3 regulates microtubules by antagonizing the depolymerization activity of stathmin. J. Cell Biol. 172, 245-257. Oikonomou, V., Moretti, S., Renga, G., Galosi, C., Borghi, M., Pariano, M., Puccetti, M., Palmerini, C.A., Amico, L., Carotti, A., et al. (2016). Noncanonical fungal autophagy inhibits inflammation in response to IFN- gamma via DAPK1. Cell Host Microbe 20, 744-757.

Pauwels, A.M., Trost, M., Beyaert, R., and Hoffmann, E. (2017). Patterns, re- ceptors, and signals: regulation of phagosome maturation. Trends Immunol 38, 407-422. Peters van Ton, A.M., Kox, M., Abdo, W.F., and Pickkers, P. (2018). Precision immunotherapy for sepsis. Front. Immunol. 9, 1926.

Quinta' s-Cardama, A., Vaddi, K., Liu, P., Manshouri, T., Li, J., Scherle, P.A., Caulder, E., Wen, X., Li, Y., Waeltz, P., et al. (2010). Preclinical characterization of the selective JAK1/2 inhibitor INCB018424: therapeutic implications for the treatment of myeloproliferative neoplasms. Blood $115,3109-3117$.

Rai, A., Pathak, D., Thakur, S., Singh, S., Dubey, A.K., and Mallik, R. (2016). Dynein clusters into lipid microdomains on phagosomes to drive rapid trans- port toward lysosomes. Cell 164, 722-734. Reinhart, K., Daniels, R., Kissoon, N., Machado, F.R., Schachter, R.D., and Finfer, S. (2017). Recognizing sepsis as a global health priority - a WHO reso- lution. N. Engl. J. Med. 377, 414-417. 
Rello, J., van Engelen, T.S.R., Alp, E., Calandra, T., Cattoir, V., Kern, W.V., Netea, M.G., Nseir, S., Opal, S.M., van de Veerdonk, F.L., et al. (2018). Towards precision medicine in sepsis: a position paper from the European so- ciety of clinical microbiology and infectious diseases. Clin. Microbiol. Infect. 24, 1264-1272.

Sanjuan, M.A., Dillon, C.P., Tait, S.W., Moshiach, S., Dorsey, F., Connell, S., Komatsu, M., Tanaka, K., Cleveland, J.L., Withoff, S., and Green, D.R. (2007). Toll-like receptor signalling in macrophages links the autophagy pathway to phagocytosis. Nature 450, 1253-1257.

Shen, Q., Beucler, M.J., Ray, S.C., and Rappleye, C.A. (2018). Macrophage activation by IFNgamma triggers restriction of phagosomal copper from intra- cellular pathogens. PLoS Pathog 14, e1007444.

Siddiquee, K., Zhang, S., Guida, W.C., Blaskovich, M.A., Greedy, B., Lawrence, H.R., Yip, M.L., Jove, R., McLaughlin, M.M., Lawrence, N.J., et al. (2007). Selective chemical probe inhibitor of Stat3, identified through structure-based virtual screening, induces antitumor activity. Proc. Natl. Acad. Sci. USA 104, 7391-7396.

Singer, M., Deutschman, C.S., Seymour, C.W., Shankar-Hari, M., Annane, D., Bauer, M., Bellomo, R., Bernard, G.R., Chiche, J.D., Coopersmith, C.M., et al. (2016). The third international consensus definitions for sepsis and septic shock (Sepsis-3). JAMA 315, 801-810.

Sokolovska, A., Becker, C.E., Ip, W.K., Rathinam, V.A., Brudner, M., Paquette, N., Tanne, A., Vanaja, S.K., Moore, K.J., Fitzgerald, K.A., et al. (2013). Activation of caspase-1 by the NLRP3 inflammasome regulates the NADPH oxidase NOX2 to control phagosome function. Nat. Immunol. 14, 543-553.

Subramanian Vignesh, K., Landero Figueroa, J.A., Porollo, A., Caruso, J.A., and Deepe, G.S., Jr. (2013). Granulocyte macrophage-colony stimulating fac- tor induced Zn sequestration enhances macrophage superoxide and limits intracellular pathogen survival. Immunity 39, 697-710.

Taylor, P.R., Roy, S., Meszaros, E.C., Sun, Y., Howell, S.J., Malemud, C.J., and Pearlman, E. (2016). JAK/STAT regulation of Aspergillus fumigatus corneal in- fections and IL-6/23stimulated neutrophil, IL-17, elastase, and MMP9 activity. J. Leukoc. Biol. 100, 213-222.

van der Poll, T., van de Veerdonk, F.L., Scicluna, B.P., and Netea, M.G. (2017). The immunopathology of sepsis and potential therapeutic targets. Nat. Rev. Immunol. 17, 407-420.

Venihaki, M., Dikkes, P., Carrigan, A., and Karalis, K.P. (2001). Corticotropin- releasing hormone regulates IL-6 expression during inflammation. J. Clin. Invest. 108, 1159-1166.

Via, L.E., Fratti, R.A., McFalone, M., Pagan-Ramos, E., Deretic, D., and Deretic, V. (1998). Effects of cytokines on mycobacterial phagosome matura- tion. J. Cell Sci. 111, 897-905.

Wen, H., Dou, Y., Hogaboam, C.M., and Kunkel, S.L. (2008). Epigenetic regu- lation of dendritic cell-derived interleukin-12 facilitates immunosuppression after a severe innate immune response. Blood 111, 1797-1804. 
Zhang, H., Rodriguez, S., Wang, L., Wang, S., Serezani, H., Kapur, R., Cardoso, A.A., and Carlesso, N. (2016). Sepsis induces hematopoietic stem cell exhaustion and myelosuppression through distinct contributions of TRIF and MYD88. Stem Cell Rep 6, 940-956. 


\section{Figure legends}

Figure 1. LAP blockade in monocytes is a hallmark of immunoparalysis in patients with sepsis. (A) Outline of the study protocol. (B) Monocytes obtained from healthy controls or patients upon sepsis recovery (day 7) were stimulated for $30 \mathrm{~min}$ with conidia of A. fumigatus melanin-deficient $\triangle p k s P$ strain and analyzed for LC3+ phagosome (LAPosome) formation. Oneway ANOVA, and Tukey's multiple comparisons. (C and D) Comparative analysis of (C) p47phox and (D) CD63 recruitment on the phagosome of monocytes stimulated as in (B). Oneway ANOVA, Tukey's multiple comparisons. (E) Correlation between LAPosome formation and clearance of Aspergillus conidia (ATCC 46645, wild-type strain) by human monocytes obtained from sepsis patients (day 7; $n=9$ ) and healthy controls $(n=5)$. Killing was assessed at $24 \mathrm{~h}$ of infection by CFU counting. Pearson correlation coefficient, two tailed. (F) IL-6 production in culture supernatants of monocytes following overnight stimulation with conidia of melanindeficient Aspergillus ( $\triangle p k s P$ strain). Student's t test. (G) Differences in Apache II score of patients with intact LAP versus defective LAP responses. Unpaired Student's t test. (H) Kaplan Mayer curves on the cumulative probability of secondary infections over time ( $\mathrm{n}=43$ patients; $\mathrm{n}=54$ infectious episodes). Log-rank test. ${ }^{* * *} \mathrm{p}<0.0001,{ }^{* *} \mathrm{p}<0.001,{ }^{*} \mathrm{p}<0.01$.

Figure 2. ERK signaling on the phagosome regulates NADPH oxidase-mediated activation of LAP. (A) Monocytes obtained from healthy individuals were left untreated or stimulated with PMA or conidia of Aspergillus $\Delta p k s P$ strain (MOI 10:1) and phosphorylation of p47phox was determined in cell lysates by immunoblot analysis. (B) Human monocytes stimulated with Aspergillus as in (A) with or without the presence of the MEK1/2 inhibitor UO126 and phosphorylation of ERK1/2 and p47phox (Ser345) was determined in cell lysates by immunoblot analysis. (C) Kinetics of ERK recruitment and representative fluorescent image of ERK localization to the phagosome in monocytes at $30 \mathrm{~min}$ of infection with Aspergillus $\Delta p k s P$ strain. Scale bar, $5 \mu \mathrm{m}$. (D and E) Data on quantification of $\mathrm{p} 47 \mathrm{phox}^{+}$(D) and p22phox ${ }^{+}$(E) phagosomes in human monocytes stimulated with Aspergillus with or without UO126 as in (B). Unpaired Student's t test. (F) Human monocytes were left untreated or stimulated with Aspergillus for $1 \mathrm{~h}$ with or without UO126, and intracellular ROS production was determined. (G) Data on quantification of $\mathrm{LC}^{+}$phagosomes in human monocytes stimulated as in (D). Unpaired Student's t test. (H) Killing of Aspergillus $\Delta p k s P$ conidia by human monocytes infected as in $\mathrm{d}$ for $24 \mathrm{~h}$ and assessed by CFU counts. Unpaired Student's t test. (I-K) (I) Data on quantification of $\mathrm{LC}^{+}(\mathrm{I})$, p47phox ${ }^{+}(\mathrm{J})$, and $\mathrm{ERK}^{+}(\mathrm{K})$ phagosomes in monocytes obtained from a healthy individual (HC) and two patients following recovery from septic shock (day 7) stimulated as in (D). Unpaired Student's t test. Representative fluorescent images of ERK localization in phagosomes are shown. ${ }^{* *} \mathrm{p}<0.0001,{ }^{* *} \mathrm{p}<0.001,{ }^{* *} \mathrm{p}<0.01$.

Figure 3. Autocrine IL-6 signaling regulates ERK trafficking to the phagosome for LAPmediated fungal killing. (A-F) Data on quantification of (A) p22phox ${ }^{+}$, (B) p47phox ${ }^{+}$, (C) LC3 $^{+}$, (D) VATPase ${ }^{+}$, (E) cathepsin-D ${ }^{+}$, and (F) FITC-Dextran ${ }^{+}$phagosomes in BMDMs from 
${\mathrm{IL}-6^{-/-}}(\mathrm{KO})$ or control IL- $6^{+/+}$mice infected with Aspergillus $\Delta p k s P$ strain for 15 min (A-C) or $1 \mathrm{~h}(\mathrm{D}-\mathrm{F})$. Unpaired Student's $\mathrm{t}$ test. (G) BMDMs from IL- $6^{-/}$(KO) or control IL- $6^{+/+}$mice were infected with conidia of Aspergillus wild-type strain (ATCC 46645) for $6 \mathrm{~h}$ and killing was assessed by counting the viability of intracellular conidia. Unpaired Student's $t$ test. (H) Fungal loads in lung homogenates from IL- $6^{-/-}(\mathrm{KO})(\mathrm{n}=9)$ or control IL- $6^{+/+}(\mathrm{n}=9)$ mice infected via intratracheal administration of wild-type Aspergillus fumigatus (ATCC 46645) assessed by CFU counts at $48 \mathrm{~h}$ of infection. Mann-Whitney test. (I) BMDMs from IL- $6^{-/-}$(KO) or control IL- $6^{+/+}$ mice were left untreated or stimulated with Aspergillus for $15 \mathrm{~min}$ and phosphorylation of ERK1/2 was determined in cell lysates by immunoblot analysis. (J) Quantification of relative density of p-ERK1/2 to total ERK1/2 in blots from four different experiment performed as in (I). Mann-Whitney test. (K) Representative fluorescent images of ERK phosphorylation (p-ERK) and ERK localization on the phagosome of BMDMs stimulated as in (J). Scale bar, $5 \mu \mathrm{m}$. (L) Data on quantification of $\mathrm{ERK}^{+}$phagosomes in BMDMs of IL-6 ${ }^{-/-}(\mathrm{KO})$, IL- ${ }^{+/+}$(control), or IL-6 (KO) mice following IL-6 supplementation in culture media upon infection with Aspergillus (30 min). One-way ANOVA, Tukey's multiple comparisons. (M) BMDMs from IL-6 $6^{-/}$(KO) or control IL- $6^{+/+}$mice were infected with conidia of Aspergillus $\Delta p k s P$ strain or wild-type strain (ATCC 46645 ) with or without supplementation of culture media with IL-6, TNF, or IFN- $\gamma$, and killing was assessed by counting the viability of intracellular conidia at the indicated time point. Oneway ANOVA, Tukey's multiple comparisons. ${ }^{* *} \mathrm{p}<0.0001,{ }^{* *} \mathrm{p}<0.001,{ }^{*} \mathrm{p}<0.01$.

Figure 4. IL-6/JAK2/ninein autocrine axis regulates microtubule-dependent ERK trafficking and LAPosome formation. (A) Data on quantification of $\mathrm{ERK}^{+}$phagosomes in BMDMs obtained from C57BL/6 (B6) mice left untreated or treated with nocodazole $(5 \mu \mathrm{M})$ or taxol $(5 \mu \mathrm{M})$. One-way ANOVA, and Tukey's multiple comparisons. (B) Representative fluorescent images of $\beta$-tubulin in uninfected BMDMs from IL- $6^{-/-}(\mathrm{KO}), \mathrm{IL}^{-} 6^{+/+}$(control), or IL$6 \mathrm{KO}$ mice following IL-6 supplementation in culture media. Scale bar, $5 \mu \mathrm{m}$. (C) Representative fluorescent images of $\beta$-tubulin in BMDMs from $\mathrm{IL}_{-} 6^{-/-}(\mathrm{KO}), \mathrm{IL}^{-6^{+/+}}$(control), or IL-6 KO mice following IL-6 supplementation, upon infection with Aspergillus $\Delta p k s P$ strain (MOI 3:1, 30 min). Scale bar, $5 \mu \mathrm{m}$. (D) Representative time-lapse images of Aspergillus phagosome transport in BMDMs of IL- $6^{+/+}$(control) or IL- $6^{-/-}(\mathrm{KO})$ mice. Scale bar, $5 \mu \mathrm{m}$. (E) Data on quantification of the transport of Aspergillus-containing phagosomes from the minus end of microtubules to the organizing center of BMDMs analyzed from the experiment performed in (C). One-way ANOVA and Tukey's multiple comparisons. ( $\mathrm{F}-\mathrm{I})$ Data on quantification of $(\mathrm{F})$ Aspergillus-containing phagosomes transport, $(\mathrm{G}) \mathrm{ERK}^{+}$phagosomes, (H) LAPosome formation, and (I) fungal killing, in BMDMs obtained from B6 mice left untreated, or treated with JAK2 inhibitor (ruxolitinib) and infected with Aspergillus $\triangle p k s P$ strain. One-way ANOVA and Tukey's multiple comparisons. (J) Data on quantification of $\gamma$-tubulin relative fluorescent intensity at the microtubule organizing center of uninfected BMDMs from IL- $6^{-/-}(\mathrm{KO})$, IL- $6^{+/+}$(control), or IL-6 KO mice following IL-6 supplementation in culture media for $15 \mathrm{~min}$. (K) Data on quantification of ninein relative fluorescent intensity at the microtubule organizing center of uninfected BMDMs from IL- $6^{-/}$ $(\mathrm{KO}), \mathrm{IL}^{+}{ }^{+/+}$(control), or IL-6 (KO) mice following IL-6 supplementation. One-way ANOVA and Tukey's multiple comparisons. (L) Representative fluorescent images of $\gamma$-tubulin from experiment performed in (J). Scale bar, $5 \mu \mathrm{m}$. (M) Representative fluorescent images of immunostaining for ninein and JAK2 in uninfected BMDMs from IL- $6^{-/-}(\mathrm{KO}), \mathrm{IL}_{-} 6^{+/+}$(control), or IL-6 (KO) mice following IL-6 supplementation. ${ }^{* * *} \mathrm{p}<0.0001,{ }^{* *} \mathrm{p}<0.001,{ }^{*} \mathrm{p}<0.01$. 
Figure 5. Increased susceptibility of mice recovering from sepsis to fungal disease is associated with LAP blockade in monocytes/macrophages. (A) Outline of the mouse model of bacterial peritonitis (CLP). (B) Survival rates of C57BL/6 (B6) mice subjected to different degree of severity of polymicrobial sepsis (CLP) or SHAM treated (control) mice. (C) Fungal loads of C57BL/6 (B6) mice infected via intratracheal administration of Aspergillus (ATCC46645 strain) following the recovery from mild, severe sepsis or SHAM treatment (day 7), assessed by CFU counts at $48 \mathrm{~h}$. Mann-Whitney test. (D) Histopathology (GMS staining) from representative tissue sections of lungs from B6 mice on day 3 of infection with Aspergillus wild-type strain as in (C) following recovery from severe sepsis (hereafter sepsis) or SHAM treatment. Magnification $\times 400$. (E) Representative immunofluorescent images of LAPosome formation in splenic monocytes from B6 and BMDMs from GFP-LC3 transgenic mice stimulated for 30 min with Aspergillus (melanin-deficient $\Delta p k s P$ strain) following recovery from severe sepsis (sepsis) or SHAM (control). (F) Data on quantification of $\mathrm{LC}^{+}$phagosomes in monocytes, BMDMs, or alveolar macrophages (AMs) stimulated as in (E). One-way ANOVA and Tukey's multiple comparisons. (G) Intracellular ROS production in BMDMs from mice following recovery from sepsis (day 7) or control SHAM treatment. (H) Assessment of phagolysosomal fusion in BMDMs pre-loaded with FITC-dextran and infected for $4 \mathrm{~h}$ as in (G). (I) Representative fluorescence images are shown in (I). Scale bar, $5 \mu \mathrm{m}$. (J-L) Data on quantification of $\mathrm{VATPase}^{+}(\mathrm{J})$, cathepsin $\mathrm{D}^{+}(\mathrm{K})$, and LAMP- ${ }^{+}(\mathrm{L})$ phagosomes in BMDMs from B6 mice stimulated as in (E). Unpaired Student's t test. (M) Data on killing of Aspergillus (ATCC 46645 strain) by BMDMs obtained from B6 mice following recovery from sepsis or SHAM treatment. Unpaired Student's t test. ${ }^{* *} \mathrm{p}<0.0001,{ }^{* *} \mathrm{p}<0.001,{ }^{*} \mathrm{p}<0.01$.

Figure 6. Restoration of IL-6 signaling defects in mice and human patients recovering from sepsis reverses LAP blockade and impaired fungal clearance. (A) Representative fluorescent images of $\beta$-tubulin in uninfected BMDMs from B6 mice following SHAM treatment or recovery from sepsis with or without IL-6 supplementation. Scale bar, $5 \mu \mathrm{m}$. (B) Data on quantification of the transport of Aspergillus-containing phagosomes ( $\triangle p k s P$ strain) in BMDMs obtained from experiments performed as in (A). One-way ANOVA and Tukey's multiple comparisons. (C) Representative images of ERK recruitment on the phagosome of BMDMs (30 min) obtained from experiments performed as in (B). Scale bar, $5 \mu \mathrm{m}$. (D and E) Data on quantification of $\mathrm{ERK}^{+}(\mathrm{D})$ and $\mathrm{LC}^{+}(\mathrm{E})$ phagosomes in BMDMs from experiment performed in (C). One-way ANOVA and Tukey's multiple comparisons. (F) BMDMs from SHAM treated mice, or mice recovering from sepsis (day 7) were infected with the indicated Aspergillus strain, with or without supplementation with IL-6, TNF, or IFN- $\gamma$. Killing of intracellular conidia was assessed by counting of viable (germinating) conidia. One-way ANOVA and Tukey's multiple comparisons. (G) Representative fluorescent images of $\beta$-tubulin in monocytes from a healthy control (HC) individual, and patients recovering from sepsis at $30 \mathrm{~min}$ of infection with Aspergillus $4 p k s P$ strain. Scale bar, $5 \mu \mathrm{m}$. (H) Monocytes obtained from sepsis patients and healthy controls (HC) were infected with conidia of Aspergillus fumigatus (ATCC 46645 strain), with or without IL-6 supplementation and killing of conidia was assessed by CFU counting at 24 h. Unpaired Student's $t$ test. ${ }^{* *} \mathrm{p}<0.0001,{ }^{* *} \mathrm{p}<0.001,{ }^{*} \mathrm{p}<0.01$. 
Figure 7. Loss of IL-6 signaling is sepsis results in increased susceptibility to bacterial and fungal pathogens, specifically via inhibiting LAP. (A) Representative immunostaining of $\beta$ tubulin in BMDMs obtained from uninfected $\mathrm{Atg} 5^{+/+}$(control) and Atg $5^{-/-}$(KO) mice. (B-D) Data on quantification of (B) FITC-Dextran ${ }^{+}$, (C) VATPase ${ }^{+}$, and (D) LAMP-1 ${ }^{+}$phagosomes in control and Atg5 KO BMDMs upon infection with $\triangle p k s P$ Aspergillus strain $(1 \mathrm{~h})$. Unpaired Student's t test. (E) Killing of Aspergillus conidia by control and Atg5 KO BMDMs assessed at 6 h. Unpaired Student's t test. (F-I) (F) BMDMs obtained from control and Atg5 KO mice following recovery (day 7) from sevesepsis) or SHAM (control) treatment were infected with Aspergillus with or without IL-6 supplementation and killing of conidia was assessed at $6 \mathrm{~h}$ oneway ANOVA and Tukey's multiple comparisons. (G) BMDMs from IL-6 KO or control mice were infected with Pseudomonas aeruginosa (ATCC 27853 strain). The number of phagocytosed bacteria at $1 \mathrm{~h}$ was assessed by CFU plating. Bacterial killing by BMDMs was assessed by CFU counts of intracellular bacteria at $12 \mathrm{~h}$ of infection. One-way ANOVA and Tukey's multiple comparisons. (H) Killing of Pseudomonas aeruginosa (ATCC 27853 strain) following infection of control and Atg5 KO BMDMs assessed as in (H). Unpaired Student's t test. (I) BMDMs obtained from control and Atg5 KO mice following recovery (day 7) from sepsis or SHAM (control) treatment were infected with Pseudomonas aeruginosa with or without IL-6 supplementation and bacterial killing was assessed as in $(\mathrm{H})$. One-way ANOVA and Tukey's multiple comparisons post hoc test. ${ }^{* * *} \mathrm{p}<0.00001,{ }^{* * *} \mathrm{p}<0.0001,{ }^{* *} \mathrm{p}<0.001,{ }^{*} \mathrm{p}<0.01$. 